\title{
DEGENERATE PARABOLIC OPERATORS OF KOLMOGOROV TYPE WITH A GEOMETRIC CONTROL CONDITION*
}

\author{
Karine Beauchard ${ }^{1}$, Bernard Helffer ${ }^{2}$, Raphael Henry ${ }^{2}$ and Luc Robbiano ${ }^{3}$
}

\begin{abstract}
We consider Kolmogorov-type equations on a rectangle domain $(x, v) \in \Omega=\mathbb{T} \times(-1,1)$, that combine diffusion in variable $v$ and transport in variable $x$ at speed $v^{\gamma}, \gamma \in \mathbb{N}^{*}$, with Dirichlet boundary conditions in $v$. We study the null controllability of this equation with a distributed control as source term, localized on a subset $\omega$ of $\Omega$. When the control acts on a horizontal strip $\omega=\mathbb{T} \times(a, b)$ with $0<a<b<1$, then the system is null controllable in any time $T>0$ when $\gamma=1$, and only in large time $T>T_{\text {min }}>0$ when $\gamma=2$ (see [K. Beauchard, Math. Control Signals Syst. 26 (2014) 145-176]). In this article, we prove that, when $\gamma>3$, the system is not null controllable (whatever $T$ is) in this configuration. This is due to the diffusion weakening produced by the first order term. When the control acts on a vertical strip $\omega=\omega_{1} \times(-1,1)$ with $\overline{\omega_{1}} \subset \mathbb{T}$, we investigate the null controllability on a toy model, where $\left(\partial_{x}, x \in \mathbb{T}\right)$ is replaced by $\left(i(-\Delta)^{1 / 2}, x \in \Omega_{1}\right)$, and $\Omega_{1}$ is an open subset of $\mathbb{R}^{N}$. As the original system, this toy model satisfies the controllability properties listed above. We prove that, for $\gamma=1,2$ and for appropriate domains $\left(\Omega_{1}, \omega_{1}\right)$, then null controllability does not hold (whatever $T>0$ is), when the control acts on a vertical strip $\omega=\omega_{1} \times(-1,1)$ with $\overline{\omega_{1}} \subset \Omega_{1}$. Thus, a geometric control condition is required for the null controllability of this toy model. This indicates that a geometric control condition may be necessary for the original model too.
\end{abstract}

Mathematics Subject Classification. 93C20, 93B05, 93B07.

Received September 13, 2013. Revised June 4, 2014.

Published online March 9, 2015.

Keywords and phrases. Null controllability, degenerate parabolic equation, hypoelliptic operator, geometric control condition.

* The authors were partially supported by the "Agence Nationale de la Recherche" (ANR), Projet Blanc EMAQS number ANR-2011-BS01-017-01 and Projet NOSEVOL number ANR-2011-BS01-019-01.

1 Centre de Mathématiques Laurent Schwartz, Ecole Polytechnique, 91128 Palaiseau cedex, France. Karine.Beauchard@math.polytechnique.fr

2 Département de Mathématiques, Batiment 425, Université Paris Sud, 91405 Orsay cedex, France.

Bernard.Helffer@math.u-psud.fr; Raphael.Henry@math.u-psud.fr

3 Laboratoire de Mathématiques de Versailles (LM-Versailles), Université de Versailles Saint-Quentin-en-Yvelines, CNRS UMR 8100, 45 Avenue des Etats-Unis, 78035 Versailles, France. luc.robbiano@uvsq.fr 


\section{INTRODUCTION}

\subsection{Origin of the problem}

The goal of this article is to study the null controllability of Kolmogorov-type equations

$$
\begin{cases}\partial_{t} f(t, x, v)-v^{\gamma} \partial_{x} f(t, x, v)-\partial_{v}^{2} f(t, x, v)=u(t, x, v) 1_{\omega}(x, v), & (t, x, v) \in(0, T) \times \Omega, \\ f(t, x, \pm 1)=0, & (t, x) \in(0, T) \times \mathbb{T}, \\ f(0, x, v)=f_{0}(x, v), & (x, v) \in \Omega,\end{cases}
$$

where $\Omega=\mathbb{T} \times(-1,1), \mathbb{T}$ is the $1 \mathrm{D}$-torus, $\gamma \in \mathbb{N}^{*}, T>0$, and the control is a source term $u(t, x, v)$ localized on a nonempty open subset $\omega$ of $\Omega$. This equation is close to linearizations of Prandtl or Crocco-type equations for fluids $[13,14,39]$; this motivates the study of the controllability of (1.1).

Definition 1.1 (null controllability). Let $T>0$ and $\gamma \in \mathbb{N}^{*}$. System (1.1) is null controllable in time $T$ if, for any $f_{0} \in L^{2}(\Omega)$, there exists $u \in L^{2}((0, T) \times \Omega)$ such that the solution of $(1.1)$ satisfies $f(T, \cdot, \cdot)=0$.

By duality, null controllability is equivalent to observability for the adjoint system

$$
\begin{cases}\partial_{t} g(t, x, v)+v^{\gamma} \partial_{x} g(t, x, v)-\partial_{v}^{2} g(t, x, v)=0, & (t, x, v) \in(0,+\infty) \times \Omega, \\ g(t, x, \pm 1)=0, & (t, x) \in(0, T) \times \mathbb{T}, \\ g(0, x, v)=g_{0}(x, v), & (x, v) \in \Omega\end{cases}
$$

Definition 1.2 (observability). Let $T>0, \gamma \in \mathbb{N}^{*}$ and $\omega$ be a non empty open subset of $\Omega$. System (1.2) is observable in $\omega$ in time $T$ if there exists $\mathcal{C}>0$ such that, for any $g_{0} \in L^{2}(\Omega)$, the solution of the Cauchy problem (1.2) satisfies

$$
\int_{\Omega}|g(T, x, v)|^{2} \mathrm{~d} x \mathrm{~d} v \leqslant \mathcal{C} \int_{0}^{T} \int_{\omega}|g(t, x, v)|^{2} \mathrm{~d} x \mathrm{~d} v \mathrm{~d} t .
$$

Equation (1.2) combines diffusion in variable $v$ and transport in variable $x$ (at speed $v^{\gamma}$ ). Thanks to the interplay between these two phenomena, the equation diffuses both in variables $v$ and $x$ (see Prop. 6.2), contrarily to equation $\left(\partial_{t}-\partial_{v}^{2}\right) g(t, x, v)=0$. But, the global diffusion is weaker than for the $2 \mathrm{D}$ heat equation $\left(\partial_{t}-\partial_{x}^{2}-\partial_{v}^{2}\right) g(t, x, v)=0$. Thus, natural questions are the following ones.

Question 1: Is the diffusion in variable $v$ strong enough for observability to hold when the control acts on a horizontal strip $\omega=\mathbb{T} \times(a, b)$ with $0<a<b<1$, whatever $\gamma \in \mathbb{N}^{*}$ is? (i.e. as for equation $\left(\partial_{t}-\partial_{v}^{2}\right) g(t, x, v)=0$, $(t, x, v) \in(0, T) \times \mathbb{T} \times(-1,1))$

Question 2: Is the diffusion in variable $x$ sufficient for null controllability to hold when the control acts on a vertical strip $\omega=\omega_{1} \times(-1,1)$ where $\omega_{1} \subset \subset \mathbb{T}$ ? (i.e. as for the $2 \mathrm{D}$ heat equation $\left(\partial_{t}-\partial_{x}^{2}-\partial_{v}^{2}\right) g(t, x, v)=0$, $(t, x, v) \in(0, T) \times \mathbb{T} \times(-1,1))$.

The goal of this article is to answer the first question and to study the second one for a toy-model.

Question 1 is studied in [9], where the following result is proved.

\section{Theorem 1.3.}

1. If $\gamma=1$ and $\omega=\mathbb{T} \times(a, b)$ with $-1<a<b<1$, then system (1.2) is observable in $\omega$ in any time $T>0$.

2. If $\gamma=2$ and $\omega=\mathbb{T} \times(a, b)$ with $0<a<b<1$ then there exists $T^{*} \geqslant a^{2} / 2$ such that

- system $(1.2)$ is observable in $\omega$ in any time $T>T^{*}$;

- system (1.2) is not observable in $\omega$ in time $T<T^{*}$.

3. If $\gamma=2$ and $\omega=\mathbb{T} \times(a, b)$ with $-1<a<0<b<1$ then system (1.2) is observable in any time $T>0$.

Statements 2 and 3 above show that, when $\gamma=2$, the information needs time to reach the degeneracy line $\{v=0\}$ from the observation location $\omega$ when $\bar{\omega} \cap\{v=0\}=\emptyset$. 


\subsection{Main results}

The first goal of this article is to prove that observability does not hold, when $\gamma \geqslant 3$ and the control acts on a horizontal strip: the presence of the first order term $v^{\gamma} \partial_{x} f$ in the equation reduces diffusion in the variable $v$ so strongly that observability becomes false. Thus, Theorems 1.3 and 1.4 below answer Question 1.

Theorem 1.4. If $\gamma \geqslant 3$ and $\omega=\mathbb{T} \times(a, b)$ with $0<a<b<1$, then system (1.2) is not observable in $\omega$ (whatever $T>0$ is).

The second goal of this article is to investigate null controllability of equation (1.2) for $\gamma \in\{1,2\}$ when the control acts on a vertical strip $\omega=\omega_{1} \times(-1,1)$ where $\omega_{1} \subset \subset \mathbb{T}$. Unfortunately, we are not able to work directly on equation (1.2). Thus, we consider the following toy model.

$$
\begin{cases}\partial_{t} g(t, x, v)+i v^{\gamma}\left(-\Delta_{x}^{D}\right)^{\beta} g(t, x, v)-\partial_{v}^{2} g(t, x, v)=0, & (t, x, v) \in(0, T) \times \Omega, \\ g(t, x, \pm 1)=0, & (t, x) \in(0, T) \times \Omega_{1}, \\ g(0, x, v)=g_{0}(x, v), & (x, v) \in \Omega_{1} \times(-1,1),\end{cases}
$$

where

- $\Omega:=\Omega_{1} \times(-1,1), \Omega_{1}$ is a bounded open subset of $\mathbb{R}^{N_{1}}$ and $N_{1} \in \mathbb{N}^{*}$;

- $\Delta_{x}^{D}$ is the Dirichlet-Laplace operator on $\Omega_{1}$

$$
D\left(\Delta_{x}^{D}\right)=H^{2} \cap H_{0}^{1}\left(\Omega_{1}\right), \quad \Delta_{x}^{D} g=\Delta g ;
$$

- $\gamma \in \mathbb{N}^{*}, \beta \in(0,1)$.

The case $\beta=1 / 2$ is of particular interest for system (1.2). The presence of " $i$ " in the term " $i v^{\gamma}\left(-\Delta_{x}^{D}\right)^{\beta} g$ " aims at ensuring the skew symmetry of this operator, as in the original model. We use the same definition for the observability of systems (1.2) and (1.3).

We are able to deny observability with explicit counterexamples, under an appropriate assumption $\mathcal{P}(s)$ on the open sets $\left(\Omega_{1}, \omega_{1}\right)$. In order to express this assumption, we introduce the non decreasing sequence $\left(\lambda_{n}\right)_{n \in \mathbb{N}^{*}}$ of the eigenvalues of $\left(-\Delta_{x}^{D}\right)$ on $\Omega_{1}$ and a corresponding orthonormal sequence of associated eigenfunctions,

$$
\left\{\begin{array}{l}
-\Delta \varphi_{n}(x)=\lambda_{n} \varphi_{n}(x), x \in \Omega_{1}, \\
\varphi_{n}(x)=0, \\
\left\|\varphi_{n}\right\|_{L^{2}\left(\Omega_{1}\right)}=1 .
\end{array}\right.
$$

Definition 1.5 (property $\mathcal{P}(s))$. Let $s \in(0,1 / 2)$ and $\omega_{1}$ be an open subset of $\Omega_{1}$. The pair $\left(\Omega_{1}, \omega_{1}\right)$ satisfies the property $\mathcal{P}(s)$ if

$$
\varlimsup_{n \rightarrow+\infty}\left[\frac{-1}{\lambda_{n}^{s}} \ln \left(\int_{\omega_{1}}\left|\varphi_{n}(x)\right|^{2} \mathrm{~d} x\right)\right]=+\infty .
$$

This assumption is related to the classical problem of high-frequency localization of the eigenfunctions of the Laplacian. Note that $1 / 2$ is the optimal upperbound for possible values of $s$ (see [35], Thm. 5.4 and Prop. 5.5). Particular examples of pairs $\left(\Omega_{1}, \omega_{1}\right)$ satisfying property $\mathcal{P}(s)$ for any $s \in(0,1 / 2)$ are discussed in Section 4 . For instance, if $\Omega_{1}$ is a conical open subset of $\mathbb{R}^{d}(d \geqslant 2)$ generated by an open subset $U$ of $\mathbb{S}^{d-1}$,

$$
\Omega_{1}=\left\{x=r x^{\prime} ; 0<r<1, x^{\prime} \in U\right\},
$$

and $\omega_{1}$ is an open subset of $\Omega_{1}$ that does not intersect its boundary $\partial \Omega_{1}$, then the pair $\left(\Omega_{1}, \omega_{1}\right)$ satisfies property $\mathcal{P}(s)$ for every $s \in(0,1 / 2)$. One can indeed construct a subsequence of eigenfunctions $\widetilde{\varphi}_{k}$ localized near the boundary $\partial \Omega_{1}$, called "whispering gallery eigenmodes".

Our first nonobservability result concerns system (1.3) for $\gamma=1$. 
Theorem 1.6. We assume $\gamma=1$.

1. If $\beta>0$ and $\omega=\Omega_{1} \times(a, b)$ where $0<a<b<1$ then system (1.3) is observable in $\omega$ in any time $T>0$.

2. If $\beta \in(0,3 / 4)$ and $\left(\Omega_{1}, \omega_{1}\right)$ satisfies property $\mathcal{P}\left(\frac{2 \beta}{3}\right)$, then system $(1.3)$ is not observable in $\omega=\omega_{1} \times(-1,1)$ (whatever $T>0$ is).

In particular, when $\beta=1 / 2$, the diffusion in the variable $v$ is strong enough for system (1.3) to be observable in a horizontal strip $\omega=\Omega_{1} \times(a, b)$ in any positive time $T$. On the contrary, the diffusion in the variable $x$ is too weak for system (1.3) to be observable in a vertical strip $\omega=\omega_{1} \times(-1,1)$ in finite time $T$, at least for appropriate pairs $\left(\Omega_{1}, \omega_{1}\right)$ that satisfy property $\mathcal{P}(1 / 3)$ (which happens, for instance, when $\Omega_{1}$ is a bounded conical open subset of $\mathbb{R}^{d}$ and $\overline{\omega_{1}} \subset \Omega_{1}$ ). Thus a geometric control condition (GCC) on $(\Omega, \omega)$ is required for system (1.3) to be observable in $\omega$. As a consequence, we conjecture that system (1.2), with $\gamma=1$, requires a GCC to be observable.

Our second noncontrollability result concerns system (1.3) for $\gamma=2$.

Theorem 1.7. We assume $\gamma=2$.

1. If $\beta>0$ and $\omega=\Omega_{1} \times(a, b)$ where $0<a<b<1$ then there exists $T^{*} \geqslant a^{2} / 2$ such that

- system (1.3) is observable in $\omega$ in any time $T>T^{*}$;

- system (1.3) is not observable in $\omega$ in time $T<T^{*}$.

2. If $\beta \in(0,1)$ and $\left(\Omega_{1}, \omega_{1}\right)$ satisfies property $\mathcal{P}\left(\frac{\beta}{2}\right)$, then system $(1.3)$ is not observable in $\omega=\omega_{1} \times(-1,1)$ (whatever $T>0$ is).

In particular, when $\beta=1 / 2$, the diffusion in the variable $v$ is strong enough for system (1.3) to be observable in a horizontal strip $\omega=\Omega_{1} \times(a, b)$, but information needs time to propagate from the observation location $\omega$ to the degeneracy set $\{v=0\}$. On the contrary, the diffusion in the variable $x$ is too weak for (1.3) to be observable in a vertical strip $\omega=\omega_{1} \times(-1,1)$ in finite time $T$, at least for appropriate pairs $\left(\Omega_{1}, \omega_{1}\right)$. Thus a GCC on $(\Omega, \omega)$ is required for (1.3) to be observable in $\omega$. As a consequence, we conjecture that system (1.2), with $\gamma=2$, requires a GCC to be observable.

\subsection{Bibliographical comments}

\subsubsection{Null controllability of the heat equation}

The null and approximate controllabilities of the heat equation are essentially well understood subjects. In particular, the heat equation on a smooth bounded domain $\Omega$ of $\mathbb{R}^{d}\left(d \in \mathbb{N}^{*}\right)$, with a source term located on an open subset $\omega$ of $\Omega$, is null controllable in arbitrarily small time $T$ and with an arbitrarily small control support $\omega$. This result is related to the infinite speed of propagation. It is proved, for the case $d=1$ by Fattorini and Russell ([24], Thm. 3.3), and, for $d \geqslant 2$ by O. Imanuvilov [31,32] (see also the book [26] by Fursikov and Imanuvilov) and Lebeau and Robbiano [34]. It is then natural to wonder whether the same result holds for degenerate parabolic equations.

\subsubsection{Boundary-degenerate parabolic equations}

The null controllability of 1D-parabolic equations degenerating on the boundary of the space domain is well understood: it still holds for weak degeneracies and fails for strong ones, see [2,15-20,25,36]. Fewer results are available for multidimensional problems, see [21].

\subsubsection{Parabolic equations degenerating inside the domain}

In [37], Martinez et al. study linearized Crocco type equations

$$
\left\{\begin{array}{lc}
\partial_{t} f(t, x, v)+\partial_{x} f(t, x, v)-\partial_{v v} f(t, x, v)=u(t, x, v) 1_{\omega}(x, v), & (t, x, v) \in(0, T) \times \mathbb{T} \times(0,1), \\
f(t, x, 0)=f(t, x, 1)=0, & (t, x) \in(0, T) \times \mathbb{T} .
\end{array}\right.
$$


For a given strict open subset $\omega$ of $\mathbb{T} \times(0,1)$, they prove that null controllability does not hold: the optimal result is regional null controllability. Note that, for Kolmogorov-type equations (1.2), the coupling between diffusion in $v$ and transport in $x$ (at speed $v^{\gamma}$ ) generates diffusion both in variables $x$ and $v$ (see Prop. 6.2). As a consequence, the controllability properties are different.

In [10], Beauchard et al. study Grushin-type equations

$$
\left\{\begin{array}{lr}
\partial_{t} f(t, x, y)-\partial_{x}^{2} f(t, x, y)-|x|^{2 \gamma} \partial_{y}^{2} f(t, x, y)=u(t, x, y) 1_{\omega}(x, y), & (t, x, y) \in(0, T) \times \Omega, \\
f(t, x, y)=0, & (t, x, y) \in(0, T) \times \partial \Omega
\end{array}\right.
$$

where $\Omega:=(-1,1) \times(0,1), \omega \subset(0,1) \times(0,1)$, and $\gamma>0$. Here, the parabolic operator degenerates along the line $\{0\} \times(0,1)$. They prove that null controllability

- holds in any time $T>0$ and any control support $\omega$ when $\gamma \in(0,1)$;

- does not hold (whatever $T$ and $\omega$ ) when $\gamma>1$;

- holds only in large time when $\gamma=1$ and $\omega=(a, b) \times(0,1)$ with $0<a<b<1$.

Note that, contrary to Grushin-type equations (1.5), in Kolmogorov-type equations (1.2), the parabolic operator degenerates everywhere on the domain.

\subsubsection{Unique continuation for Kolmogorov-type equations}

In this section, we focus on unique continuation for Kolmogorov-type equations (1.2), i.e. whether the property $g(t, x, v) \equiv 0$ on $(0, T) \times \omega$ does imply $g \equiv 0$ on $(0, T) \times \Omega$, for a given open subset $\omega$ of $\Omega$.

When $\omega=\mathbb{T} \times(a, b)$ is an horizontal strip, then the unique continuation of equation (1.2) holds for every $\gamma \in$ $\mathbb{N}^{*}$, as a consequence of Holmgren's theorem (the coefficients of the operator are analytic and the hypersurface $\mathbb{T} \times\{a, b\}$ is noncharacteristic). In particular, Theorem 1.4 emphasizes that, when $\gamma \geqslant 3$, then observability does not hold even if unique continuation holds.

To our best knowledge, when $\omega$ is a general open subset of $\Omega$, then unique continuation for Kolmogorov-type equations (1.2) is an open problem.

Bony proved in [11] that Hörmander's operators of the form $P=\sum_{j} X_{j}^{2}$ (i.e. such that the Lie algebra generated by the $X_{j}$ has maximal rank at any point) with analytic coefficients, satisfy the unique continuation, in the following sense: if, for some $f$ with non zero gradient, $f^{-1}(a)$ is a strongly noncharacteristic surface and $u$ is a distribution such that $P u=0$ and $u=0$ on $f^{-1}[(-\infty, a)]$, then $u \equiv 0$ on a neighborhood of $f^{-1}(a)$. The validity of the same result for Hörmander's operators of the form $P=X_{0}+\sum_{j} X_{j}^{2}$ (generalizing our Kolmogorov operator $\mathcal{K}=\partial_{t}+v^{\gamma} \partial_{x}-\partial_{v}^{2}$ ) is an open problem.

When coefficients are not analytic, but only $C^{\infty}$, unique continuation may not hold. For instance, Alinhac and Zuily built in [3] a zero order $C^{\infty}$-perturbation of the Kolmogorov operator $\mathcal{K}=\partial_{t}+v^{\gamma} \partial_{x}-\partial_{v}^{2}$ for which unique continuation does not hold. There exist $C^{\infty}$-functions $u(t, x, v)$ and $a(t, x, v)$ on a neighborhood $V$ of 0 in $\mathbb{R}^{3}$ such that $\mathcal{K} u+a u=0, u(t, x, v)=a(t, x, v)=0$ when $v<0$, and $0 \in \operatorname{Supp}(u)$. And the same result holds with any surface $\{v=$ const. $\}$.

The result of Alinhac and Zuily leaves open the question of the unique continuation for system (1.2). Indeed, their counterexample does not satisfy the boundary conditions of (1.2) and it cannot be built with $a=0$. However, it suggests that unique continuation for system (1.2) is a subtle issue.

\subsection{Structure of the paper}

The article is organized as follows.

Section 2 is devoted to the proof of Theorem 1.4.

In Section 3, we prove the negative statements of Theorems 1.6 and 1.7. These results rely on a fine semi classical analysis of the complex Airy and Davies operators.

In Section 4 , we propose examples of pairs $\left(\Omega_{1}, \omega_{1}\right)$ satisfying property $\mathcal{P}(s)$ for any $s \in(0,1 / 2)$. 
The proof of the positive results of Theorems 1.6 and 1.7 relies on the decomposition of the solution of (1.3) on a Hilbert basis of $L^{2}\left(\Omega_{1}\right)$, called 'Fourier decomposition' with a slight abuse of vocabulary. Thus, the validity of this decomposition and associated well-posedness results are treated in Section 5 .

In Section 6, we prove the positive results of Theorems 1.6 and 1.7. The strategy is the same as in [9], but intermediate results have been improved. Hence we rewrite the proof completely. First, we state a Carleman estimate for the 1D-heat equation satisfied by the Fourier components. Then, we quantify the dissipation of Fourier modes; this result is stronger than in [9]. Then, we combine these two tools to prove the first statements of Theorems 1.6 and 1.7 .

\section{NONOBSERVABILITY WHEN $\gamma \geqslant 3$}

The goal of this section is the proof of Theorem 1.4. The strategy is the same as in Section 5.3 of [9], but intermediate results are different. Let $\gamma \in \mathbb{N}^{*}, a, b, T \in \mathbb{R}$ be fixed, in the whole section, such that

$$
\gamma \geqslant 3, T>0 \text { and } 0<a<b<1 \text {. }
$$

Step 1: Approximate solution.

Let $\epsilon>0$ be such that $b<1-\epsilon$ and $\theta_{ \pm} \in C^{\infty}(\mathbb{R})$ be such that $\operatorname{Supp}\left(\theta_{-}\right) \subset(-1-\epsilon,-1+\epsilon), \operatorname{Supp}\left(\theta_{+}\right) \subset$ $(1-\epsilon, 1+\epsilon)$ and $\theta_{ \pm}( \pm 1)=1$. Let $\mu \in \mathbb{C}$ be some eigenvalue, with smallest real part, of the operator $\left(-\partial_{y}^{2}+i y^{\gamma}\right)$, with domain

$$
\mathcal{D}_{\gamma}:=\left\{u \in H^{2}(\mathbb{R}) \text { s. t. } y^{\gamma} u \in L^{2}(\mathbb{R})\right\} .
$$

Note that this operator has compact resolvent (see [28]); moreover, $\mu$ is a simple eigenvalue and a real number if $\gamma=3$. Let $\xi$ be an associated eigenfunction

$$
\left\{\begin{array}{l}
-\xi^{\prime \prime}(y)+i y^{\gamma} \xi(y)=\mu \xi(y), y \in \mathbb{R} \\
\|\xi\|_{L^{2}(\mathbb{R})}=1
\end{array}\right.
$$

We recall that (see [42], Chap. 10, Sects. 59 and 60)

$$
|\xi(y)| \leqslant C \mathrm{e}^{-c|y|^{\frac{2+\gamma}{2}}}, \quad \forall y \in \mathbb{R}
$$

for some constants $C, c>0$.

For $n \in \mathbb{N}^{*}$, we define

$$
\tilde{g}_{n}(t, v):=n^{\frac{1}{2(2+\gamma)}}\left[\xi\left(n^{\frac{1}{2+\gamma}} v\right)-\sum_{\sigma \in\{-,+\}} \xi\left(\sigma n^{\frac{1}{2+\gamma}}\right) \theta_{\sigma}(v)\right] \mathrm{e}^{-\mu n^{\frac{2}{2+\gamma}} t} .
$$

We have

$$
\begin{cases}\partial_{t} \tilde{g}_{n}(t, v)+i n v^{\gamma} \tilde{g}_{n}(t, v)-\partial_{v}^{2} \tilde{g}_{n}(t, v)=E_{n}(t, v), & (t, v) \in(0, T) \times(-1,1), \\ \tilde{g}_{n}(t, \pm 1)=0, & t \in(0, T),\end{cases}
$$

where

$$
E_{n}(t, v)=n^{\frac{1}{2(2+\gamma)}} \sum_{\sigma \in\{-,+\}}\left(\left(\mu n^{\frac{2}{2+\gamma}}-i n v^{\gamma}\right) \theta_{\sigma}(v)+\theta_{\sigma}^{\prime \prime}(v)\right) \xi\left(\sigma n^{\frac{1}{2+\gamma}}\right) \mathrm{e}^{-\mu n^{\frac{2}{2+\gamma}} t} .
$$

Let $g_{n}$ be the solution of

$$
\begin{cases}\partial_{t} g_{n}(t, v)+i n v^{\gamma} g_{n}(t, v)-\partial_{v}^{2} g_{n}(t, v)=0, & (t, v) \in(0, T) \times(-1,1), \\ g_{n}(t, \pm 1)=0, & t \in(0, T), \\ g_{n}(0, v)=\tilde{g}_{n}(0, v), & v \in(-1,1) .\end{cases}
$$


We have

$$
\frac{1}{2} \frac{\mathrm{d}}{\mathrm{d} t}\left\|\left(\tilde{g}_{n}-g_{n}\right)(t)\right\|_{L^{2}(-1,1)}^{2}=-\left\|\partial_{v}\left(\tilde{g}_{n}-g_{n}\right)(t)\right\|_{L^{2}(-1,1)}^{2}+\operatorname{Re}\left(\int_{-1}^{1} \overline{E_{n}(t, v)}\left(\tilde{g}_{n}-g_{n}\right)(t, v) \mathrm{d} v\right)
$$

By Poincaré and Cauchy-Schwarz Inequalities, we deduce that, for every $t \in[0, T]$,

$$
\frac{\mathrm{d}}{\mathrm{d} t}\left\|\left(\tilde{g}_{n}-g_{n}\right)(t)\right\|_{L^{2}(-1,1)}^{2} \leqslant-\frac{\pi^{2}}{4}\left\|\left(\tilde{g}_{n}-g_{n}\right)(t)\right\|_{L^{2}(-1,1)}^{2}+\frac{4}{\pi^{2}}\left\|E_{n}(t)\right\|_{L^{2}(-1,1)}^{2} .
$$

From this inequality and (2.2), we deduce that, for every $t \in[0, T]$

$$
\begin{aligned}
\left\|\left(\tilde{g}_{n}-g_{n}\right)(t)\right\|_{L^{2}(-1,1)}^{2} & \leqslant \frac{4}{\pi^{2}} \int_{0}^{t}\left\|E_{n}(\tau)\right\|_{L^{2}(-1,1)}^{2} \mathrm{e}^{-\frac{\pi^{2}}{4}(t-\tau)} \mathrm{d} \tau \\
& \leqslant C n^{2+\frac{1}{2+\gamma}} \sum_{\sigma \in\{-1,1\}}\left|\xi\left(\sigma n^{\frac{1}{2+\gamma}}\right)\right|^{2} \int_{0}^{t} \mathrm{e}^{\left(-2 \operatorname{Re}(\mu) n^{\frac{2}{2+\gamma}+\frac{\pi^{2}}{4}}\right) \tau} \mathrm{d} \tau \\
& \leqslant C n^{2-\frac{1}{2+\gamma}} \sum_{\sigma \in\{-1,1\}}\left|\xi\left(\sigma n^{\frac{1}{2+\gamma}}\right)\right|^{2}
\end{aligned}
$$

where the constant $C$ may change from line to line.

By (2.1), we deduce that

$$
\left\|\left(\tilde{g}_{n}-g_{n}\right)(t)\right\|_{L^{2}(-1,1)} \leqslant C n^{\frac{3+2 \gamma}{2(2+\gamma)}} \mathrm{e}^{-c \sqrt{n}}, \quad \forall t \in[0, T] .
$$

Step 2: Conclusion.

Working by contradiction, we assume that system (1.2) is observable in $\omega$ in time $T$. The observability inequality applied to the solution $g(t, x, v):=g_{n}(t, v) \mathrm{e}^{i n x}$ of $(1.2)$ gives

$$
\int_{-1}^{1}\left|g_{n}(T, v)\right|^{2} \mathrm{~d} v \leqslant \mathcal{C} \int_{0}^{T} \int_{a}^{b}\left|g_{n}(t, v)\right|^{2} \mathrm{~d} v \mathrm{~d} t, \quad \forall n \in \mathbb{N}^{*} .
$$

We deduce from the triangular inequality, the previous relation and (2.3) that

$$
\begin{aligned}
\left\|\tilde{g}_{n}(T)\right\|_{L^{2}(-1,1)} \leqslant & \left(\mathcal{C} \int_{0}^{T} \int_{a}^{b}\left|\tilde{g}_{n}(t, v)\right|^{2} \mathrm{~d} v \mathrm{~d} t\right)^{1 / 2}+\left\|\left(\tilde{g}_{n}-g_{n}\right)(T)\right\|_{L^{2}(-1,1)} \\
& +\left(\mathcal{C} \int_{0}^{T} \int_{a}^{b}\left|\left(\tilde{g}_{n}-g_{n}\right)(t, v)\right|^{2} \mathrm{~d} v \mathrm{~d} t\right)^{1 / 2} \\
& \leqslant\left(\mathcal{C} \int_{0}^{T} \int_{a}^{b}\left|\tilde{g}_{n}(t, v)\right|^{2} \mathrm{~d} v \mathrm{~d} t\right)^{1 / 2}+(1+\sqrt{T \mathcal{C}}) C n^{\frac{3+2 \gamma}{2(2+\gamma)}} \mathrm{e}^{-c \sqrt{n}} .
\end{aligned}
$$

However, there exists $C>0$ such that

$$
\left\|\tilde{g}_{n}(T)\right\|_{L^{2}} \geqslant C \mathrm{e}^{-\operatorname{Re}(\mu) n^{\frac{2}{2+\gamma}} T}
$$


and

$$
\begin{aligned}
\left(\int_{0}^{T} \int_{a}^{b}\left|\tilde{g}_{n}(t, v)\right|^{2} \mathrm{~d} v \mathrm{~d} t\right)^{1 / 2} & =\left(\int_{0}^{T} \int_{a}^{b} n^{\frac{1}{(2+\gamma)}}\left|\xi\left(n^{\frac{1}{2+\gamma}} v\right)\right|^{2} \mathrm{e}^{-2 \operatorname{Re}(\mu) n^{\frac{2}{2+\gamma}} t} \mathrm{~d} v \mathrm{~d} t\right)^{1 / 2} \text { because } b<1-\epsilon \\
& =\left(\int_{a n^{\frac{1}{2+\gamma}}}^{b n^{\frac{1}{2+\gamma}}}|\xi(y)|^{2} \mathrm{~d} y\right)^{1 / 2}\left(\int_{0}^{T} \mathrm{e}^{-2 \operatorname{Re}(\mu) n^{\frac{2}{2+\gamma}} t} \mathrm{~d} t\right)^{1 / 2} \\
& \leqslant C n^{\frac{-1}{2+\gamma}}\left(\int_{a n^{\frac{1}{2+\gamma}}}^{b n^{\frac{1}{2+\gamma}}} \mathrm{e}^{-2 c|y|^{\frac{2+\gamma}{2}}} \mathrm{~d} y\right)^{1 / 2} \text { by }(2.1) \\
& \leqslant C n^{\frac{-1}{2(2+\gamma)}} \mathrm{e}^{-c a^{\frac{2+\gamma}{2}} \sqrt{n}} .
\end{aligned}
$$

This gives a contradiction, when $n \rightarrow+\infty$, because $\frac{2}{2+\gamma}<\frac{1}{2}$ when $\gamma>2$.

\section{NONOBSERVABILITY ON A VERTICAL STRIP}

The goal of this section is the proof of the nonobservability results of Theorems 1.6 and 1.7.

\subsection{Accurate spectral analysis}

In this section, we are interested in the spectrum of the operators

$$
\mathcal{A}_{(-R, R)}:=-\frac{\mathrm{d}^{2}}{\mathrm{~d} y^{2}}+i y \quad \text { and } \quad \mathcal{H}_{(-R, R)}:=-\frac{\mathrm{d}^{2}}{\mathrm{~d} y^{2}}+i y^{2}
$$

defined on the segment $(-R, R), R>0$, with Dirichlet boundary conditions at $y= \pm R$, with domains

$$
\mathcal{D}\left(\mathcal{A}_{(-R, R)}\right)=\mathcal{D}\left(\mathcal{H}_{(-R, R)}\right)=H^{2} \cap H_{0}^{1}((-R, R), \mathbb{C}) .
$$

More precisely, we study the asymptotic behavior, as $R \rightarrow+\infty$, of the bottom of the spectrum of $\mathcal{A}_{(-R, R)}$ and $\mathcal{H}_{(-R, R)}$ and we prove the following two theorems, in Sections 3.3 and 3.4, respectively.

Theorem 3.1. Let $\mu_{1}<0$ be the first zero of the Airy function. Then,

$$
\lim _{R \rightarrow \infty}\left(\inf \operatorname{Re} \sigma\left(\mathcal{A}_{(-R, R)}\right)\right)=\frac{\left|\mu_{1}\right|}{2},
$$

where $\sigma\left(\mathcal{A}_{(-R, R)}\right)$ denotes the spectrum of $\mathcal{A}_{(-R, R)}$. Moreover, for every $\varepsilon>0$, there exists $R_{\varepsilon}>0$ and $M_{\varepsilon}>0$ such that, for every $R \geq R_{\varepsilon}$,

$$
\sup _{\substack{\gamma \leq \mu_{1} \mid / 2-\varepsilon \\ \nu \in \mathbb{R}}}\left\|\left(\mathcal{A}_{(-R, R)}-(\gamma+i \nu)\right)^{-1}\right\|_{\mathcal{L}\left(L^{2}(-R, R)\right)} \leqslant M_{\varepsilon} .
$$

Now, let us consider the case of the Davies operator.

Theorem 3.2. We have

$$
\lim _{R \rightarrow \infty}\left(\inf \operatorname{Re} \sigma\left(\mathcal{H}_{(-R, R)}\right)\right)=\frac{\sqrt{2}}{2} .
$$

Moreover, for every $\varepsilon>0$, there exists $R_{\varepsilon}^{\prime}>0$ and $M_{\varepsilon}^{\prime}>0$ such that, for every $R \geq R_{\varepsilon}^{\prime}$,

$$
\sup _{\gamma \leq \sqrt{2} / 2-\varepsilon}\left\|\left(\mathcal{H}_{(-R, R)}-(\gamma+i \nu)\right)^{-1}\right\|_{\mathcal{L}\left(L^{2}(-R, R)\right)} \leqslant M_{\varepsilon}^{\prime} .
$$

Analogous questions have been considered in [4-8] in relation with problems occuring in superconductivity. We study these two operators using the techniques developed in these references. The study of more general cases (dimension 2) complementary to those studied in $[4,5]$ will be done in [30]. 


\subsection{Proof of the negative statements of Theorems 1.6 and 1.7}

The goal of this subsection is the proof of the second statements of Theorems 1.6 and 1.7, by application of the results of the previous subsection. Thus, in the whole subsection, $\gamma, \beta, \Omega_{1}$ and $\omega_{1}$ are fixed such that

- either $\gamma=1, \beta \in(0,3 / 4)$ and $\left(\Omega_{1}, \omega_{1}\right)$ satisfies property $\mathcal{P}(2 \beta / 3)$;

- or $\gamma=2, \beta \in(0,1)$ and $\left(\Omega_{1}, \omega_{1}\right)$ satisfies property $\mathcal{P}(\beta / 2)$.

For $n \in \mathbb{N}^{*}$, we introduce the operator $A_{n, \gamma}$ defined by

$$
D\left(A_{n, \gamma}\right):=H^{2} \cap H_{0}^{1}((-1,1), \mathbb{C}), \quad A_{n, \gamma} \psi:=-\frac{\mathrm{d}^{2} \psi}{\mathrm{d} v^{2}}+i \lambda_{n}^{\beta} v^{\gamma} \psi
$$

By rescaling $\left(y=\lambda_{n}^{\frac{\beta}{2+\gamma}} v\right)$ and using Theorems 3.1 and 3.2, there exist $\mathcal{C}_{1}, \mathcal{C}_{2}>0$ and $n_{*} \in \mathbb{N}^{*}$ such that, for every $n \geqslant n_{*}, A_{n, \gamma}$ has an eigenvalue $\mu_{n}$ satisfying

$$
\mathcal{C}_{1} \lambda_{n}^{\frac{2 \beta}{2+\gamma}} \leqslant \operatorname{Re}\left(\mu_{n}\right) \leqslant \mathcal{C}_{2} \lambda_{n}^{\frac{2 \beta}{2+\gamma}}
$$

We introduce a normalized eigenfunction $\psi_{n}$ of $A_{n, \gamma}$ associated with the eigenvalue $\mu_{n}$,

$$
\left\{\begin{array}{l}
-\psi_{n}^{\prime \prime}(v)+i \lambda_{n}^{\beta} v^{\gamma} \psi_{n}(v)=\mu_{n} \psi_{n}(v), v \in(-1,1), \\
\psi_{n}( \pm 1)=0 \\
\left\|\psi_{n}\right\|_{L^{2}(-1,1)}=1 .
\end{array}\right.
$$

Then the function

$$
g_{n}(t, x, v):=\varphi_{n}(x) \psi_{n}(v) \mathrm{e}^{-\mu_{n} t}
$$

is a solution of (1.3). The second statement of Theorems 1.6 and 1.7 is a consequence of the following proposition.

Proposition 3.3. For every $T>0$, we have

$$
\varliminf_{n \rightarrow+\infty}\left(\frac{\int_{0}^{T} \int_{\omega}\left|g_{n}(t, x, v)\right|^{2} \mathrm{~d} x \mathrm{~d} v \mathrm{~d} t}{\int_{\Omega}\left|g_{n}(T, x, v)\right|^{2} \mathrm{~d} x \mathrm{~d} v}\right)=0 .
$$

Proof of Proposition 3.3.

We have

$$
\int_{\Omega}\left|g_{n}(T, x, v)\right|^{2} \mathrm{~d} v=\mathrm{e}^{-2 \operatorname{Re}\left(\mu_{n}\right) T},
$$

because $\psi_{n}$ and $\varphi_{n}$ are normalized in $L^{2}$.

By Fubini's Theorem, we get

$$
\begin{aligned}
\int_{0}^{T} \int_{\omega}\left|g_{n}(t, x, v)\right|^{2} \mathrm{~d} x \mathrm{~d} v \mathrm{~d} t & =\left(\int_{0}^{T} \mathrm{e}^{-2 \operatorname{Re}\left(\mu_{n}\right) t} \mathrm{~d} t\right)\left(\int_{-1}^{1}\left|\psi_{n}(v)\right|^{2} \mathrm{~d} v\right)\left(\int_{\omega_{1}}\left|\varphi_{n}(x)\right|^{2} \mathrm{~d} x\right) \\
& =\frac{1-\mathrm{e}^{-2 \operatorname{Re}\left(\mu_{n}\right) T}}{2 \operatorname{Re}\left(\mu_{n}\right)} \int_{\omega_{1}}\left|\varphi_{n}(x)\right|^{2} \mathrm{~d} x .
\end{aligned}
$$

Thus,

$$
\frac{\int_{0}^{T} \int_{\omega}\left|g_{n}(t, x, v)\right|^{2} \mathrm{~d} x \mathrm{~d} v \mathrm{~d} t}{\int_{\Omega}\left|g_{n}(T, x, v)\right|^{2} \mathrm{~d} x \mathrm{~d} v}=\frac{\mathrm{e}^{2 \operatorname{Re}\left(\mu_{n}\right) T}-1}{2 \operatorname{Re}\left(\mu_{n}\right)} \int_{\omega_{1}} \varphi_{n}(x)^{2} \mathrm{~d} x .
$$


Let $C$ be a positive constant such that

$$
C>2 \mathcal{C}_{2} T
$$

where $\mathcal{C}_{2}$ is as in (3.5).

Let $s:=\frac{2 \beta}{2+\gamma}$. By property $\mathcal{P}(s)$, there exists a subsequence $\left(n_{k}\right)_{k \in \mathbb{N}}$ such that

$$
\frac{-1}{\lambda_{n_{k}}^{s}} \ln \left(\int_{\omega_{1}}\left|\varphi_{n_{k}}(x)\right|^{2} \mathrm{~d} x\right) \geqslant C, \quad \forall k \in \mathbb{N},
$$

or, equivalently

$$
\int_{\omega_{1}}\left|\varphi_{n_{k}}(x)\right|^{2} \mathrm{~d} x \leqslant \mathrm{e}^{-C \lambda_{n_{k}}^{s}}, \quad \forall k \in \mathbb{N}
$$

Then,

$$
\frac{\int_{0}^{T} \int_{\omega}\left|g_{n_{k}}(t, x, v)\right|^{2} \mathrm{~d} x \mathrm{~d} v \mathrm{~d} t}{\int_{\Omega}\left|g_{n_{k}}(T, x, v)\right|^{2} \mathrm{~d} x \mathrm{~d} v} \leqslant \frac{\mathrm{e}^{\left(2 \mathcal{C}_{2} T-C\right) \lambda_{n_{k}}^{s}}}{2 \mathcal{C}_{1} \lambda_{n_{k}}^{s}} \underset{k \rightarrow+\infty}{\longrightarrow} 0,
$$

by (3.6), which gives the conclusion.

\subsection{Semi classical analysis of the complex Airy operator $(\gamma=1)$}

The goal of this subsection is the proof of Theorem 3.1.

We introduce two model-operators, that have well known spectral and pseudospectral behavior. Let $\mathcal{A}_{(-R,+\infty)}$ and $\mathcal{A}_{(-\infty, R)}$ be the Dirichlet realizations of the operator $-\frac{\mathrm{d}^{2}}{\mathrm{~d} y^{2}}+i y$ on the intervals $(-R,+\infty)$ and $(-\infty, R)$ respectively. We are going to approximate the resolvent of $\mathcal{A}_{(-R, R)}$ by the one of $\mathcal{A}_{(-R,+\infty)}$ or $\mathcal{A}_{(-\infty, R)}$ depending on where we are, respectively close to $-R$ or close to $+R$.

Let us remark that, if

$$
T_{R}: u(x) \mapsto u(x+R) \quad \text { and } \quad U_{R}: u(x) \mapsto u(R-x)
$$

then

$$
\begin{aligned}
T_{R}^{-1}\left(\mathcal{A}_{(-R,+\infty)}-\lambda\right) T_{R} & =\mathcal{A}_{(0,+\infty)}-(\lambda+i R), \\
U_{R}^{-1}\left(\mathcal{A}_{(-\infty, R)}-\lambda\right) U_{R} & =\mathcal{A}_{(0,+\infty)}^{*}-(\lambda-i R),
\end{aligned}
$$

thus

$$
\inf \operatorname{Re} \sigma\left(\mathcal{A}_{(-R, \infty)}\right)=\inf \operatorname{Re} \sigma\left(\mathcal{A}_{(-\infty, R)}\right)=\frac{\left|\mu_{1}\right|}{2}
$$

because $\inf \operatorname{Re} \sigma\left(\mathcal{A}_{(0,+\infty)}\right)=\left|\mu_{1}\right| / 2$, see [4].

Step 1. We prove

$$
\varliminf_{R \rightarrow+\infty}\left(\inf \operatorname{Re} \sigma\left(\mathcal{A}_{(-R, R)}\right)\right) \geqslant \frac{\left|\mu_{1}\right|}{2}
$$

and (3.2).

Let $\varepsilon>0$. We search $R_{\varepsilon}>0$ such that

$$
\left.\left.\forall R \geq R_{\varepsilon}, \quad \sigma\left(\mathcal{A}_{(-R, R)}\right) \cap(]-\infty,\left|\mu_{1}\right| / 2-\varepsilon\right]+i \mathbb{R}\right)=\emptyset .
$$

We recall that, by [27], there exists $C_{\varepsilon}>0$ such that

$$
\begin{aligned}
& \sup _{\substack{\gamma \leq\left|\mu_{1}\right| / 2-\varepsilon \\
\nu \in \mathbb{R}}}\left\|\left(\mathcal{A}_{(0,+\infty)}-(\gamma+i \nu)\right)^{-1}\right\|_{\mathcal{L}\left(L^{2}(0,+\infty)\right)} \leqslant C_{\varepsilon}, \\
& \sup _{\substack{\gamma \leq \mu_{1} \mid / 2-\varepsilon \\
\nu \in \mathbb{R}}}\left\|\left(\mathcal{A}_{(0,+\infty)}^{*}-(\gamma+i \nu)\right)^{-1}\right\|_{\mathcal{L}\left(L^{2}(0,+\infty)\right)} \leqslant C_{\varepsilon} .
\end{aligned}
$$


Let $\left.\lambda=\gamma+i \nu \in]-\infty,\left|\mu_{1}\right| / 2-\varepsilon\right]+i \mathbb{R}$ and $h_{+}, h_{-} \in \mathcal{C}^{\infty}(\mathbb{R} ;[0,1])$ be such that

$$
\begin{array}{rlrl}
\operatorname{Supp}\left(h_{-}\right) \subset(-\infty, 1 / 2), & h_{-} & \equiv 1 \text { on }(-\infty,-1 / 2], \\
\operatorname{Supp}\left(h_{+}\right) \subset(-1 / 2,+\infty), & h_{+} & \equiv 1 \text { on }[1 / 2,+\infty), \\
h_{-}^{2}+h_{+}^{2} & \equiv 1 \text { on }(-\infty,+\infty) .
\end{array}
$$

For $R>0$, we define

$$
\eta_{R}^{ \pm}(x)=h_{ \pm}\left(\frac{x}{R}\right) \mathbf{1}_{(-R, R)}(x)
$$

and

$$
\mathcal{R}_{R}(\lambda)=\eta_{R}^{-}\left(\mathcal{A}_{(-R,+\infty)}-\lambda\right)^{-1} \eta_{R}^{-}+\eta_{R}^{+}\left(\mathcal{A}_{(-\infty, R)}-\lambda\right)^{-1} \eta_{R}^{+} .
$$

$\mathcal{R}_{R}(\lambda)$ will be used as an approximation of the resolvent of $\mathcal{A}_{(-R, R)}$. We have

$$
\begin{aligned}
\left(\mathcal{A}_{(-R, R)}-\lambda\right) \mathcal{R}_{R}(\lambda)= & I+\left[\mathcal{A}_{(-R, R)}, \eta_{R}^{-}\right]\left(\mathcal{A}_{(-R,+\infty)}-\lambda\right)^{-1} \eta_{R}^{-} \\
& +\left[\mathcal{A}_{(-R, R)}, \eta_{R}^{+}\right]\left(\mathcal{A}_{(-\infty, R)}-\lambda\right)^{-1} \eta_{R}^{+}
\end{aligned}
$$

as an equality between operators on $L^{2}(-R, R)$.

We estimate the second term on the right hand side. In what follows, the estimates are uniform with respect to $\nu=\operatorname{Im} \lambda$. We have

$$
\left[\mathcal{A}_{(-R, R)}, \eta_{R}^{-}\right]\left(\mathcal{A}_{(-R,+\infty)}-\lambda\right)^{-1} \eta_{R}^{-}=\left(-\left(\eta_{R}^{-}\right)^{\prime \prime}-2\left(\eta_{R}^{-}\right)^{\prime} \frac{\mathrm{d}}{\mathrm{d} y}\right)\left(\mathcal{A}_{(-R,+\infty)}-\lambda\right)^{-1} \eta_{R}^{-} .
$$

Using $\left\|\left(\eta_{R}^{-}\right)^{\prime}\right\|_{L^{\infty}(-R, R)}=\mathcal{O}\left(R^{-1}\right)$ and $\left\|\left(\eta_{R}^{-}\right)^{\prime \prime}\right\|_{L^{\infty}(-R, R)}=\mathcal{O}\left(R^{-2}\right)$, we get, by (3.8) and (3.13),

$$
\left\|\left(\eta_{R}^{-}\right)^{\prime \prime}\left(\mathcal{A}_{(-R,+\infty)}-\lambda\right)^{-1} \eta_{R}^{-}\right\|_{\mathcal{L}\left(L^{2}(-R, R)\right)}=\mathcal{O}\left(\frac{1}{R^{2}}\right)
$$

Moreover, for every $v \in L^{2}(-R,+\infty)$,

$$
\left\|\frac{\mathrm{d}}{\mathrm{d} y}\left(\mathcal{A}_{(-R,+\infty)}-\lambda\right)^{-1} v\right\|_{L^{2}(-R,+\infty)} \leq\left(\left\|\left(\mathcal{A}_{(-R,+\infty)}-\lambda\right)^{-1}\right\|^{1 / 2}+\sqrt{\gamma}\left\|\left(\mathcal{A}_{(-R,+\infty)}-\lambda\right)^{-1}\right\|\right)\|v\|_{L^{2}(-R,+\infty)} .
$$

Indeed, let $w:=\left(\mathcal{A}_{(-R,+\infty)}-\lambda\right)^{-1} v$, i.e.

$$
\left\{\begin{array}{l}
-w^{\prime \prime}(y)+i y w(y)-\lambda w(y)=v(y), y \in(-R,+\infty), \\
w(-R)=w(+\infty)=0 .
\end{array}\right.
$$

We have

$$
\begin{aligned}
\left\|w^{\prime}\right\|_{L^{2}(-R,+\infty)}^{2} & =-\operatorname{Re}\left(\int_{-R}^{+\infty} \overline{w(y)} w^{\prime \prime}(y) \mathrm{d} y\right) \\
& =\operatorname{Re}\left(\int_{-R}^{+\infty} \bar{w}[i y w+\lambda w+v]\right) \\
& =\gamma \int_{-R}^{+\infty}|w|^{2}+\operatorname{Re}\left(\int_{-R}^{+\infty} \bar{w} v\right) \\
& \leqslant \gamma\|w\|_{L^{2}(-R,+\infty)}^{2}+\|w\|_{L^{2}(-R,+\infty)}\|v\|_{L^{2}(-R,+\infty)} .
\end{aligned}
$$


By taking the square root of this inequality, we get

$$
\left.\left\|w^{\prime}\right\|_{L^{2}(-R,+\infty)} \leqslant \sqrt{\gamma}\|w\|_{L^{2}(-R,+\infty)}+\|w\|_{L^{2}(-R,+\infty)}^{1 / 2}\|v\|_{L^{2}(-R,+\infty)}^{1 / 2}\right)
$$

which proves (3.20). By applying (3.20) to $v=\eta_{R}^{-} u, u \in L^{2}(\mathbb{R})$, we get

$$
\left\|\left(\eta_{R}^{-}\right)^{\prime} \frac{\mathrm{d}}{\mathrm{d} y}\left(\mathcal{A}_{(-R,+\infty)}-\lambda\right)^{-1} \eta_{R}^{-}\right\|_{\mathcal{L}\left(L^{2}(-R, R)\right)}=\mathcal{O}\left(\frac{1}{R}\right),
$$

which gives, with (3.18) and (3.19),

$$
\left\|\left[\mathcal{A}_{(-R, R)}, \eta_{R}^{-}\right]\left(\mathcal{A}_{(-R,+\infty)}-\lambda\right)^{-1} \eta_{R}^{-}\right\|_{\mathcal{L}\left(L^{2}(-R, R)\right)}=\mathcal{O}\left(\frac{1}{R}\right) .
$$

In the same way, we verify that

$$
\left\|\left[\mathcal{A}_{(-R, R)}, \eta_{R}^{+}\right]\left(\mathcal{A}_{(-\infty, R)}-\lambda\right)^{-1} \eta_{R}^{+}\right\|_{\mathcal{L}\left(L^{2}(-R, R)\right)}=\mathcal{O}\left(\frac{1}{R}\right) .
$$

Equality (3.17) can be written

$$
\left(\mathcal{A}_{(-R, R)}-\lambda\right) \mathcal{R}_{R}(\lambda)=I+\mathcal{E}_{R}(\lambda)
$$

with $\left\|\mathcal{E}_{R}(\lambda)\right\|_{\mathcal{L}\left(L^{2}(-R, R)\right)}=\mathcal{O}\left(R^{-1}\right)$, uniformly with respect to $\left.\left.\lambda \in\right]-\infty,\left|\mu_{1}\right| / 2-\varepsilon\right]+i \mathbb{R}$. We deduce the existence of $R_{\varepsilon}>0$ such that, for every $R \geq R_{\varepsilon},\left(\mathcal{A}_{(-R, R)}-\lambda\right)$ is invertible, with inverse

$$
\left(\mathcal{A}_{(-R, R)}-\lambda\right)^{-1}=\mathcal{R}_{R}(\lambda)\left(I+\mathcal{E}_{R}(\lambda)\right)^{-1} .
$$

We have proved (3.12). Moreover, according to the definition (3.16) of $\mathcal{R}_{R}(\lambda),(3.8),(3.9),(3.13)$ and (3.14) yield the estimate (3.2).

Step 2. We prove that

$$
\varlimsup_{R \rightarrow+\infty}\left(\inf \operatorname{Re} \sigma\left(\mathcal{A}_{(-R, R)}\right)\right) \leqslant \frac{\left|\mu_{1}\right|}{2} .
$$

First, we reduce the study to the complex Airy operator $\mathcal{A}_{(0, R)}$ on the interval $(0, R)$. Indeed, applying the translation $T_{R}: u(x) \mapsto u(x+R)$, we get

$$
T_{R}^{-1}\left(\mathcal{A}_{(-R, R)}-\lambda\right) T_{R}=\mathcal{A}_{(0,2 R)}-(\lambda+i R),
$$

thus $\operatorname{Re} \sigma\left(\mathcal{A}_{(-R, R)}\right)=\operatorname{Re} \sigma\left(\mathcal{A}_{(0,2 R)}\right)$. Therefore, in order to prove (3.24), we are going to prove that

$$
\varlimsup_{R \rightarrow+\infty}\left(\inf \operatorname{Re} \sigma\left(\mathcal{A}_{(0, R)}\right)\right) \leqslant \frac{\left|\mu_{1}\right|}{2} .
$$

Let $\theta_{1}, \theta_{2} \in \mathcal{C}^{\infty}(\mathbb{R} ;[0,1])$ be such that

$$
\begin{array}{rr}
\text { Supp }\left(\theta_{1}\right) \subset(-\infty, 2 / 3), & \theta_{1} \equiv 1 \text { on }(-\infty, 1 / 2), \\
\operatorname{Supp}\left(\theta_{2}\right) \subset(1 / 2,+\infty), & \theta_{2} \equiv 1 \text { on }(2 / 3,+\infty), \\
& \theta_{1}^{2}+\theta_{2}^{2} \equiv 1 \text { on } \mathbb{R} .
\end{array}
$$

For $j=1,2$ and $R>0$, we define

$$
\chi_{R}^{j}(x)=\theta_{j}\left(\frac{x}{R}\right) \mathbf{1}_{(0, R)}(x) .
$$


We want to prove that

$$
\mathbf{1}_{(0, R)}\left(\mathcal{A}_{(0, R)}+1\right)^{-1} \mathbf{1}_{(0, R)} \underset{R \rightarrow+\infty}{\longrightarrow}\left(\mathcal{A}_{(0,+\infty)}+1\right)^{-1} \quad \text { in } \mathcal{L}\left(L^{2}\left(\mathbb{R}^{+}\right)\right) .
$$

Let us remark that

$$
\sigma\left(\mathbf{1}_{(0, R)}\left(\mathcal{A}_{(0, R)}+1\right)^{-1} \mathbf{1}_{(0, R)}\right)=\sigma\left(\left(\mathcal{A}_{(0, R)}+1\right)^{-1}\right)
$$

with non vanishing eigenvalues that have the same multiplicity for both operators.

Step 2a. We prove that

$$
\mathbf{1}_{(0, R)}\left(\mathcal{A}_{(0, R)}+1\right)^{-1} \mathbf{1}_{(0, R)}-\chi_{R}^{1}\left(\mathcal{A}_{(0,+\infty)}+1\right)^{-1} \chi_{R}^{1} \underset{R \rightarrow+\infty}{\longrightarrow} 0 \quad \text { in } \mathcal{L}\left(L^{2}\left(\mathbb{R}^{+}\right)\right) .
$$

For this, we use the following approximations of the resolvent of $\left(\mathcal{A}_{(0, R)}+1\right)$,

$$
\tilde{\mathcal{R}}_{R}=\chi_{R}^{1}\left(\mathcal{A}_{(0,+\infty)}+1\right)^{-1} \chi_{R}^{1}+\chi_{R}^{2}\left(\mathcal{A}_{(0, R)}+1\right)^{-1} \chi_{R}^{2} .
$$

Then, we have

$$
\begin{aligned}
\left(\mathcal{A}_{(0, R)}+1\right) \tilde{\mathcal{R}}_{R}= & I+\left[\mathcal{A}_{(0, R)}+1, \chi_{R}^{1}\right]\left(\mathcal{A}_{(0,+\infty)}+1\right)^{-1} \chi_{R}^{1} \\
& +\left[\mathcal{A}_{(0, R)}+1, \chi_{R}^{2}\right]\left(\mathcal{A}_{(0,2 R)}+1\right)^{-1} \chi_{R}^{2},
\end{aligned}
$$

thus, by composing on the left by $\mathbf{1}_{(0, R)}\left(\mathcal{A}_{(0, R)}+1\right)^{-1} \mathbf{1}_{(0, R)}$, we get

$$
\begin{aligned}
\mathbf{1}_{(0, R)}\left(\mathcal{A}_{(0, R)}+1\right)^{-1} \mathbf{1}_{(0, R)}-\chi_{R}^{1}\left(\mathcal{A}_{(0,+\infty)}+1\right)^{-1} \chi_{R}^{1}= & \chi_{R}^{2}\left(\mathcal{A}_{(0, R)}+1\right)^{-1} \chi_{R}^{2} \\
& -\mathbf{1}_{(0, R)}\left(\mathcal{A}_{(0, R)}+1\right)^{-1} \mathbf{1}_{(0, R)}\left[\mathcal{A}_{(0, R)}+1, \chi_{R}^{1}\right] \\
& \times\left(\mathcal{A}_{(0,+\infty)}+1\right)^{-1} \chi_{R}^{1}-\mathbf{1}_{(0, R)}\left(\mathcal{A}_{(0, R)}+1\right)^{-1} \\
& \times \mathbf{1}_{(0, R)}\left[\mathcal{A}_{(0, R)}+1, \chi_{R}^{2}\right]\left(\mathcal{A}_{(0, R)}+1\right)^{-1} \chi_{R}^{2} .
\end{aligned}
$$

Now, we control the different terms on the right hand side. The terms involving commutators can be estimated as in Step 1, thanks to (3.2), and we get

$$
\begin{gathered}
\left\|\mathbf{1}_{(0, R)}\left(\mathcal{A}_{(0, R)}+1\right)^{-1} \mathbf{1}_{(0, R)}\left[\mathcal{A}_{(0, R)}+1, \chi_{R}^{1}\right]\left(\mathcal{A}_{(0,+\infty)}+1\right)^{-1} \chi_{R}^{1}\right\|_{\mathcal{L}\left(L^{2}\left(\mathbb{R}^{+}\right)\right)}=\mathcal{O}\left(\frac{1}{R}\right), \\
\left.\left\|\mathbf{1}_{(0, R)}\left(\mathcal{A}_{(0, R)}+1\right)^{-1} \mathbf{1}_{(0, R)}\left[\mathcal{A}_{(0, R)}+1, \chi_{R}^{2}\right]\left(\mathcal{A}_{(0, R)}+1\right)^{-1} \chi_{R}^{2}\right\|\right|_{\mathcal{L}\left(L^{2}\left(\mathbb{R}^{+}\right)\right)}=\mathcal{O}\left(\frac{1}{R}\right) .
\end{gathered}
$$

Moreover, for $u \in L^{2}((0, R), \mathbb{C})$, we have

$$
\operatorname{Im}\left\langle\left(\mathcal{A}_{(0, R)}+1\right) u, u\right\rangle=\langle y u, u\rangle
$$

where $\langle.,$.$\rangle denotes the L^{2}((0, R), \mathbb{C})$-hermitian product.

This relation, applied to $u=\chi_{R}^{2}\left(\mathcal{A}_{(0, R)}+1\right)^{-1} \chi_{R}^{2} f, f \in L^{2}(0,+\infty)$, which is supported in $(R / 2, R)$, gives

$$
\operatorname{Im}\left\langle\left(\mathcal{A}_{(0, R)}+1\right) u, u\right\rangle \geqslant \frac{R}{2}\|u\|^{2} .
$$

Moreover,

$$
\left(\mathcal{A}_{(0, R)}+1\right) u=\left(\chi_{R}^{2}\right)^{2} f+\left[\mathcal{A}_{(0, R)}+1, \chi_{R}^{2}\right]\left(\mathcal{A}_{(0, R)}+1\right)^{-1} \chi_{R}^{2} f .
$$


Thus, estimating the commutator as in Step 1, we get

$$
\left|\operatorname{Im}\left\langle\left(\mathcal{A}_{(0, R)}+1\right) u, u\right\rangle\right| \leqslant C\left(1+\frac{1}{R}\right)\|f\|\|u\| .
$$

Therefore,

$$
\frac{R}{2}\|u\|^{2} \leqslant C\left(1+\frac{1}{R}\right)\|f\|\|u\|
$$

We have proved that

$$
\left\|\chi_{R}^{2}\left(\mathcal{A}_{(0, R)}+1\right)^{-1} \chi_{R}^{2}\right\|_{\mathcal{L}\left(L^{2}(0,+\infty)\right)}=\mathcal{O}\left(\frac{1}{R}\right) .
$$

By (3.28), (3.29), (3.30) and (3.32), we have

$$
\left\|\mathbf{1}_{(0, R)}\left(\mathcal{A}_{(0, R)}+1\right)^{-1} \mathbf{1}_{(0, R)}-\chi_{R}^{1}\left(\mathcal{A}_{(0,+\infty)}+1\right)^{-1} \chi_{R}^{1}\right\|_{\mathcal{L}_{\left(L^{2}(0,+\infty)\right)}}=\mathcal{O}\left(\frac{1}{R}\right)
$$

which ends Step 2a.

Step 2 b. We verify that

$$
\chi_{R}^{1}\left(\mathcal{A}_{(0,+\infty)}+1\right)^{-1} \chi_{R}^{1} \underset{R \rightarrow+\infty}{\longrightarrow}\left(\mathcal{A}_{(0,+\infty)}+1\right)^{-1} \quad \text { in } \mathcal{L}\left(L^{2}(0,+\infty)\right),
$$

which ends the proof of (3.27).

To simplify notation, let us introduce

$$
\mathcal{A}_{+}=\mathcal{A}_{(0,+\infty)}+1
$$

First, we write

$$
\chi_{R}^{1} \mathcal{A}_{+}^{-1} \chi_{R}^{1} \mathcal{A}_{+}=\left(\chi_{R}^{1}\right)^{2}-\chi_{R}^{1} \mathcal{A}_{+}^{-1}\left[\mathcal{A}_{+}, \chi_{R}^{1}\right]
$$

then, composing on the right by $\mathcal{A}_{+}^{-1}$ and using that $\left(\chi_{R}^{1}\right)^{2}=1-\left(\chi_{R}^{2}\right)^{2}$,

$$
\mathcal{A}_{+}^{-1}-\chi_{R}^{1} \mathcal{A}_{+}^{-1} \chi_{R}^{1}=\left(\chi_{R}^{2}\right)^{2} \mathcal{A}_{+}^{-1}+\chi_{R}^{1} \mathcal{A}_{+}^{-1}\left[\mathcal{A}_{+}, \chi_{R}^{1}\right] \mathcal{A}_{+}^{-1} .
$$

The term involving a commutator can be estimated as in Step 1,

$$
\left\|\chi_{R}^{1} \mathcal{A}_{+}^{-1}\left[\mathcal{A}_{+}, \chi_{R}^{1}\right] \mathcal{A}_{+}^{-1}\right\|_{\mathcal{L}\left(L^{2}\left(\mathbb{R}^{+}\right)\right)}=\mathcal{O}\left(\frac{1}{R}\right)
$$

For $f \in L^{2}(0,+\infty)$, we have

$$
\begin{aligned}
\frac{R}{2}\left\|\left(\chi_{R}^{2}\right)^{2} \mathcal{A}_{+}^{-1} f\right\|^{2} & \leqslant\left\|y^{1 / 2}\left(\chi_{R}^{2}\right)^{2} \mathcal{A}_{+}^{-1} f\right\|^{2} \quad\left(\text { because Supp }\left(\chi_{R}^{2}\right) \subset(R / 2, R)\right) \\
& =\operatorname{Im}\left\langle\mathcal{A}_{+}\left(\chi_{R}^{2}\right)^{2} \mathcal{A}_{+}^{-1} f,\left(\chi_{R}^{2}\right)^{2} \mathcal{A}_{+}^{-1} f\right\rangle \\
& \leqslant\left\|\mathcal{A}_{+}\left(\chi_{R}^{2}\right)^{2} \mathcal{A}_{+}^{-1} f\right\|\left\|\left(\chi_{R}^{2}\right)^{2} \mathcal{A}_{+}^{-1} f\right\| \\
& \leqslant\left(\left\|\left(\chi_{R}^{2}\right)^{2} f\right\|+\left\|\left[\mathcal{A}_{+},\left(\chi_{R}^{2}\right)^{2}\right] \mathcal{A}_{+}^{-1} f\right\|\right)\left\|\left(\chi_{R}^{2}\right)^{2} \mathcal{A}_{+}^{-1} f\right\|,
\end{aligned}
$$

where $\langle.,$.$\rangle denotes the L^{2}((0,+\infty), \mathbb{C})$-hermitian product and $\|$.$\| is the associated norm. Estimating the term$ with a commutator as in Step 1, we get

$$
R\left\|\left(\chi_{R}^{2}\right)^{2} \mathcal{A}_{+}^{-1} f\right\|_{L^{2}(0,+\infty)} \leqslant C\left(1+\frac{1}{R}\right)\|f\|_{L^{2}(0,+\infty)} .
$$


Thus

$$
\left\|\left(\chi_{R}^{2}\right)^{2} \mathcal{A}_{+}^{-1}\right\|_{\mathcal{L}\left(L^{2}(0,+\infty)\right)}=\mathcal{O}\left(\frac{1}{R}\right) .
$$

Finally, (3.35), (3.36) and (3.37) imply (3.34).

Step 2c. Conclusion:

Step 2a and Step 2b prove (3.27). The eigenvalues of $\mathcal{A}_{+}^{-1}$ are isolated, thus we can apply ([33], Sect. IV, Paragr. 3.5). For any subsequence $R_{j} \rightarrow+\infty$ and any eigenvalue $\lambda \in \sigma\left(\mathcal{A}_{+}^{-1}\right) \backslash\{0\}$, there exists a sequence $\left(\lambda_{j}\right)$ such that, for every $j$ large enough

$$
\lambda_{j} \in \sigma\left(\mathbf{1}_{\left(0, R_{j}\right)}\left(\mathcal{A}_{\left(0, R_{j}\right)}+1\right)^{-1} \mathbf{1}_{\left(0, R_{j}\right)}\right) \backslash\{0\}=\sigma\left(\left(\mathcal{A}_{\left(0, R_{j}\right)}+1\right)^{-1}\right) \backslash\{0\}
$$

and $\lambda_{j} \rightarrow \lambda$ when $j \rightarrow+\infty$.

In particular, with $\lambda=1 /(\tilde{\lambda}+1)$, where $\tilde{\lambda}=\mathrm{e}^{i \pi / 3}\left|\mu_{1}\right| \in \sigma\left(\mathcal{A}_{(0,+\infty)}\right)$ is the eigenvalue of $\mathcal{A}_{(0,+\infty)}$ with smallest real part (see [4]), we get a sequence $\tilde{\lambda}_{j}=1 / \lambda_{j}-1 \in \sigma\left(\mathcal{A}_{\left(0, R_{j}\right)}\right)$ such that $\operatorname{Re} \tilde{\lambda}_{j} \rightarrow \operatorname{Re} \tilde{\lambda}=\left|\mu_{1}\right| / 2$, from which we deduce (3.24).

\subsection{Semi classical analysis of the Davies operator $(\gamma=2)$}

The goal of this section is the proof of Theorem 3.2, which is similar to the one of Theorem 3.1.

Step 1. Let $\varepsilon>0$. We search $R_{\varepsilon}>0$ such that

$$
\forall R \geq R_{\varepsilon}, \quad \sigma\left(\mathcal{H}_{(-R, R)}\right) \cap((-\infty, \sqrt{2} / 2-\varepsilon)+i \mathbb{R})=\emptyset
$$

and we prove (3.4).

Let $\alpha \in(0,1 / 3)$ and $\zeta_{R}^{1}, \zeta_{R}^{2}, \zeta_{R}^{3} \in \mathcal{C}^{\infty}(\mathbb{R} ;[0,1])$ be such that

$$
\begin{aligned}
\operatorname{Supp} \zeta_{R}^{1} \subset\left(-\infty,-R+R^{\alpha}\right), & \zeta_{R}^{1} \equiv 1 \text { on }\left(-\infty,-R+R^{\alpha} / 2\right), \\
\operatorname{Supp} \zeta_{R}^{2} \subset\left(-R+R^{\alpha} / 2, R-R^{\alpha} / 2\right), & \zeta_{R}^{2} \equiv 1 \text { on }\left(-R+R^{\alpha}, R-R^{\alpha}\right), \\
\operatorname{Supp} \zeta_{R}^{3} \subset\left(R-R^{\alpha},+\infty\right), & \zeta_{R}^{3} \equiv 1 \text { on }\left(R-R^{\alpha} / 2,+\infty\right), \\
\left(\zeta_{R}^{1}\right)^{2}+\left(\zeta_{R}^{2}\right)^{2}+\left(\zeta_{R}^{3}\right)^{2} \equiv 1 \text { on } \mathbb{R}, & \\
\left\|\left(\zeta_{R}^{j}\right)^{\prime}\right\|_{L^{\infty}(\mathbb{R})}=\underset{R \rightarrow+\infty}{\mathcal{O}}\left(R^{-\alpha}\right), & \left\|\left(\zeta_{R}^{j}\right)^{\prime \prime}\right\|_{L^{\infty}(\mathbb{R})}=\underset{R \rightarrow+\infty}{\mathcal{O}}\left(R^{-2 \alpha}\right) .
\end{aligned}
$$

Close to $y=-R$, we have

$$
y^{2}=-2 R(y+R)+R^{2}+o(|y+R|) .
$$

Thus, we are going to approximate $\mathcal{H}_{(-R, R)}$, close to $y=-R$, by the complex Airy type operator on $(-R,+\infty)$

$$
\mathcal{A}_{R}^{-}:=-\frac{\mathrm{d}^{2}}{\mathrm{~d} y^{2}}-2 i R(y+R)+i R^{2} .
$$

In the same way, we will approximate $\mathcal{H}_{(-R, R)}$ close to $y=+R$ by the complex Airy type operator on $(-\infty,+R)$

$$
\mathcal{A}_{R}^{+}:=-\frac{\mathrm{d}^{2}}{\mathrm{~d} y^{2}}-2 i R(R-y)+i R^{2} .
$$

Then, we remark that, if $T_{R}$ and $U_{R}$ are defined by (3.7), then we have

$$
\mathcal{A}_{R}^{-}=T_{R} \tilde{\mathcal{A}}_{2 R}^{*} T_{R}^{-1}+i R^{2} \quad \text { and } \quad \mathcal{A}_{R}^{+}=U_{R} \tilde{\mathcal{A}}_{2 R}^{*} U_{R}^{-1}+i R^{2},
$$

where $\tilde{\mathcal{A}}_{R}$ is the Dirichlet realization of the complex Airy operator $-\frac{\mathrm{d}^{2}}{\mathrm{~d} y^{2}}+i R y$ on $(0,+\infty)$. 
Following [27], we deduce that

$$
\inf \operatorname{Re} \sigma\left(\mathcal{A}_{R}^{+}\right)=\inf \operatorname{Re} \sigma\left(\mathcal{A}_{R}^{-}\right)=(2 R)^{2 / 3} \frac{\left|\mu_{1}\right|}{2},
$$

and, for every $\varepsilon>0$, there exists $C_{\varepsilon}>0$ such that

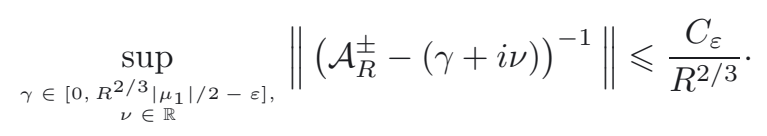

We call $\mathcal{H}_{0}$ the complex harmonic oscillator $-\frac{\mathrm{d}^{2}}{\mathrm{~d} y^{2}}+i y^{2}$ on $\mathbb{R}$, that will serve to approximate $\mathcal{H}_{(-R, R)}$ on the support of $\zeta_{R}^{2}$. We recall that $\inf \operatorname{Re} \sigma\left(\mathcal{H}_{0}\right)=\cos \pi / 4=\sqrt{2} / 2$ (see [22]) and

$$
\sup _{\substack{\gamma \leq \sqrt{2} / 2-\varepsilon \\ \nu \in \mathbb{R}}}\left\|\left(\mathcal{H}_{0}-(\gamma+i \nu)\right)^{-1}\right\| \leqslant C_{\varepsilon}^{\prime},
$$

for some $C_{\varepsilon}^{\prime}>0$, see for instance [41].

Now, we take $\lambda=\gamma+i \nu \in(0, \sqrt{2} / 2-\varepsilon)+i \mathbb{R}$ and we set

$$
\mathcal{Q}_{R}(\lambda)=\zeta_{R}^{1}\left(\mathcal{A}_{R}^{-}-\lambda\right)^{-1} \zeta_{R}^{1}+\zeta_{R}^{2}\left(\mathcal{H}_{0}-\lambda\right)^{-1} \zeta_{R}^{2}+\zeta_{R}^{3}\left(\mathcal{A}_{R}^{+}-\lambda\right)^{-1} \zeta_{R}^{3}
$$

Then, we have

$$
\begin{aligned}
\left(\mathcal{H}_{(-R, R)}-\lambda\right) \mathcal{Q}_{R}(\lambda)= & I+\left[\mathcal{H}_{(-R, R)}, \zeta_{R}^{1}\right]\left(\mathcal{A}_{R}^{-}-\lambda\right)^{-1} \zeta_{R}^{1} \\
& +\left[\mathcal{H}_{(-R, R)}, \zeta_{R}^{2}\right]\left(\mathcal{H}_{0}-\lambda\right)^{-1} \zeta_{R}^{2}+\left[\mathcal{H}_{(-R, R)}, \zeta_{R}^{3}\right]\left(\mathcal{A}_{R}^{+}-\lambda\right)^{-1} \zeta_{R}^{3} \\
& +\zeta_{R}^{1}\left(\mathcal{H}_{(-R, R)}-\mathcal{A}_{R}^{-}\right)\left(\mathcal{A}_{R}^{-}-\lambda\right)^{-1} \zeta_{R}^{1}+\zeta_{R}^{3}\left(\mathcal{H}_{(-R, R)}-\mathcal{A}_{R}^{+}\right)\left(\mathcal{A}_{R}^{+}-\lambda\right)^{-1} \zeta_{R}^{3},
\end{aligned}
$$

as equality between operators on $L^{2}(-R, R)$. The terms involving commutators can be estimated as in Step 1 of the previous section, by using (3.39), (3.41), (3.42) and we get

$$
\begin{aligned}
\left\|\left[\mathcal{H}_{(-R, R)}, \zeta_{R}^{1}\right]\left(\mathcal{A}_{R}^{-}-\lambda\right)^{-1} \zeta_{R}^{1}\right\|_{\mathcal{L}\left(L^{2}(-R, R)\right)}+\left\|\left[\mathcal{H}_{(-R, R)}, \zeta_{R}^{2}\right]\left(\mathcal{H}_{0}-\lambda\right)^{-1} \zeta_{R}^{2}\right\|_{\mathcal{L}\left(L^{2}(-R, R)\right)} & \\
& +\left\|\left[\mathcal{H}_{(-R, R)}, \zeta_{R}^{3}\right]\left(\mathcal{A}_{R}^{+}-\lambda\right)^{-1} \zeta_{R}^{3}\right\|_{\mathcal{L}\left(L^{2}(-R, R)\right)}=\mathcal{O}\left(R^{-\alpha}\right) .
\end{aligned}
$$

Moreover, we have, by definition of $\mathcal{A}_{R}^{-}$,

$$
\left(\mathcal{H}_{(-R, R)}-\mathcal{A}_{R}^{-}\right) u(y)=i(y+R)^{2} u(y),
$$

and on the support of $\zeta_{R}^{1}$, we have $y+R \leq R^{\alpha}$. Therefore, by (3.41)

$$
\begin{aligned}
\left\|\zeta_{R}^{1}\left(\mathcal{H}_{(-R, R)}-\mathcal{A}_{R}^{-}\right)\left(\mathcal{A}_{R}^{-}-\lambda\right)^{-1} \zeta_{R}^{1}\right\|_{\mathcal{L}\left(L^{2}(-R, R)\right)} & \leq R^{2 \alpha}\left\|\left(\mathcal{A}_{R}^{-}-\lambda\right)^{-1}\right\|_{\mathcal{L}\left(L^{2}(-R,+\infty)\right)} \\
& \leq C_{\varepsilon} R^{2(\alpha-1 / 3)}
\end{aligned}
$$

In the same way, we verify

$$
\left\|\zeta_{R}^{3}\left(\mathcal{H}_{(-R, R)}-\mathcal{A}_{R}^{+}\right)\left(\mathcal{A}_{R}^{+}-\lambda\right)^{-1} \zeta_{R}^{3}\right\|_{\mathcal{L}\left(L^{2}(-R, R)\right)} \leq C_{\varepsilon} R^{2(\alpha-1 / 3)} .
$$


Thus, we have proved that

$$
\left(\mathcal{H}_{(-R, R)}-\lambda\right) \mathcal{Q}_{R}(\lambda)=I+\tilde{\mathcal{E}}_{R}(\lambda),
$$

with $\left\|\tilde{\mathcal{E}}_{R}(\lambda)\right\| \rightarrow 0$ as $R \rightarrow+\infty$, uniformly with respect to $\lambda$ in the interval $(0, \sqrt{2} / 2-\varepsilon)+i \mathbb{R}$. Thus, there exists $R_{\varepsilon}>0$ such that, for every $R \geq R_{\varepsilon},\left(\mathcal{H}_{(-R, R)}-\lambda\right)$ is invertible, with

$$
\left(\mathcal{H}_{(-R, R)}-\lambda\right)^{-1}=\mathcal{Q}_{R}(\lambda)\left(I+\tilde{\mathcal{E}}_{R}(\lambda)\right)^{-1} .
$$

This proves the existence of $R_{\epsilon}>0$ such that (3.38) holds. The resolvent estimate (3.4) follows from (3.41), (3.42) and (3.43).

Step 2. We prove

$$
\varlimsup_{R \rightarrow+\infty} \inf \operatorname{Re} \sigma\left(\mathcal{H}_{(-R, R)}\right) \leqslant \frac{\sqrt{2}}{2} .
$$

Let $\varphi_{R}^{1}, \varphi_{R}^{2} \in C^{\infty}(\mathbb{R},[0,1])$ be such that

$$
\begin{aligned}
& \operatorname{Supp}\left(\varphi_{R}^{1}\right) \subset(-\infty,-R / 2) \cup(R / 2,+\infty), \varphi_{R}^{1} \equiv 1 \text { on }(-\infty,-2 R / 3) \cup(2 R / 3,+\infty), \\
& \operatorname{Supp}\left(\varphi_{R}^{2}\right) \subset(-2 R / 3,2 R / 3), \quad \varphi_{R}^{2} \equiv 1 \text { on }(-R / 2, R / 2), \\
&\left(\varphi_{R}^{1}\right)^{2}+\left(\varphi_{R}^{2}\right)^{2} \equiv 1 \text { on } \mathbb{R}, \\
&\left\|\left(\varphi_{R}^{j}\right)^{\prime}\right\|_{L^{\infty}(\mathbb{R})}=O\left(R^{-1}\right), \quad\left\|\left(\varphi_{R}^{j}\right)^{\prime \prime}\right\|_{L^{\infty}(\mathbb{R})}=O\left(R^{-2}\right) .
\end{aligned}
$$

We recall that $\mathcal{H}_{0}$ denotes the operator $-\frac{\mathrm{d}^{2}}{\mathrm{~d} x^{2}}+i x^{2}$ defined on $\mathbb{R}$, and we set

$$
\tilde{\mathcal{Q}}_{R}=\varphi_{R}^{2}\left(\mathcal{H}_{0}+1\right)^{-1} \varphi_{R}^{2}+\varphi_{R}^{1}\left(\mathcal{H}_{(-R, R)}+1\right)^{-1} \varphi_{R}^{1} .
$$

Thus, we have

$$
\left(\mathcal{H}_{(-R, R)}+1\right) \tilde{\mathcal{Q}}_{R}=I+\mathcal{P}_{R},
$$

where

$$
\mathcal{P}_{R}=\left[\mathcal{H}_{(-R, R)}, \varphi_{R}^{2}\right]\left(\mathcal{H}_{0}+1\right)^{-1} \varphi_{R}^{2}+\left[\mathcal{H}_{(-R, R)}, \varphi_{R}^{1}\right]\left(\mathcal{H}_{(-R, R)}+1\right)^{-1} \varphi_{R}^{1},
$$

and

$$
\left\|\mathcal{P}_{R}\right\|_{\mathcal{L}\left(L^{2}(-R, R)\right)}=\mathcal{O}\left(R^{-1}\right) .
$$

By composing on the left with $\left(\mathcal{H}_{(-R, R)}+1\right)^{-1}$, we get

$$
\left(\mathcal{H}_{(-R, R)}+1\right)^{-1}-\varphi_{R}^{2}\left(\mathcal{H}_{0}+1\right)^{-1} \varphi_{R}^{2}=\varphi_{R}^{1}\left(\mathcal{H}_{(-R, R)}+1\right)^{-1} \varphi_{R}^{1}-\left(\mathcal{H}_{(-R, R)}+1\right)^{-1} \mathcal{P}_{R}
$$

By going back over the proof of (3.32) and replacing (3.31) by

$$
\operatorname{Im}\left\langle\mathcal{H}_{(-R, R)} u, u\right\rangle=\left\langle x^{2} u, u\right\rangle,
$$

we get

$$
\left\|\varphi_{R}^{1}\left(\mathcal{H}_{(-R, R)}+1\right)^{-1} \varphi_{R}^{1}\right\|_{\mathcal{L}\left(L^{2}(-R, R)\right)}=\mathcal{O}\left(\frac{1}{R}\right) .
$$

By (3.47), the previous relation, together with (3.46) and (3.4) imply

$$
\left\|\left(\mathcal{H}_{(-R, R)}+1\right)^{-1}-\varphi_{R}^{2}\left(\mathcal{H}_{0}+1\right)^{-1} \varphi_{R}^{2}\right\|_{\mathcal{L}\left(L^{2}(-R, R)\right)}=\mathcal{O}\left(\frac{1}{R}\right) .
$$

Then, we prove that the operator $\varphi_{R}^{2}\left(\mathcal{H}_{0}+1\right)^{-1} \varphi_{R}^{2}$ converges to $\left(\mathcal{H}_{0}+1\right)^{-1}$ in $\mathcal{L}\left(L^{2}(\mathbb{R})\right)$, when $R \rightarrow+\infty$, with the same arguments as in Step $2 \mathrm{~b}$ of the previous section. Thus, (3.45) is proved, with the same arguments as in Step 2c of the previous section, and this ends the proof of Theorem 3.2. 


\section{EXAmples of $\left(\Omega_{1}, \omega_{1}\right)$ SATISFying PROPERTy $\mathcal{P}(\mathbf{s})$}

The goal of this section is to give examples of pairs $\left(\Omega_{1}, \omega_{1}\right)$ that satisfy property $\mathcal{P}(s)$ for any $s \in(0,1 / 2)$. Precisely, we prove that it is the case if $\Omega_{1}$ is a conical bounded subset of $\mathbb{R}^{d}$ and $\omega_{1}$ is any open subset of $\Omega_{1}$ that does not intersect the boundary $\partial \Omega_{1}$. Note that the result covers the situation where $\Omega_{1}$ is a disk or a circular sector in $2 \mathrm{D}$, a ball in any space dimension.

Proposition 4.1. Let $d \in \mathbb{N}, d \geqslant 2$ and $U$ be an open subset of $\mathbb{S}^{d-1}$. Let $\Omega_{1}$ be the conical open subset of $\mathbb{R}^{d}$ defined by

$$
\Omega_{1}:=\left\{x=r x^{\prime} ; 0<r<1, x^{\prime} \in U\right\} .
$$

Let $\omega_{1}$ be an open subset compactly embedded in $\Omega_{1}$. There exist constants $C, K>0$, a sequence $\left(\widetilde{\lambda}_{k}\right)_{k \in \mathbb{N}^{*}}$ of eigenvalues of the operator $\left(-\Delta_{\Omega_{1}}^{D}\right)$ (with domain $H^{2} \cap H_{0}^{1}\left(\Omega_{1}\right)$ ) and associated normalized eigenvectors $\left(\widetilde{\varphi}_{k}\right)_{k \in \mathbb{N}^{*}}$ such that

$$
\int_{\omega_{1}}\left|\widetilde{\varphi}_{k}(x)\right|^{2} \mathrm{~d} x \leqslant K \mathrm{e}^{-C \sqrt{\tilde{\lambda}_{k}}}, \quad \forall k \in \mathbb{N}^{*} .
$$

In particular $\left(\Omega_{1}, \omega_{1}\right)$ satisfies property $\mathcal{P}(s)$ for any $s \in(0,1 / 2)$.

We refer to [38] for other similar results. Our Proof of Proposition 4.1 relies on properties of Bessel functions, recalled in the next statement.

Proposition 4.2. The Bessel functions of the first kind $J_{\nu}$ satisfy

$$
\begin{aligned}
0 & <J_{\nu}(\nu x) \leqslant \mathrm{e}^{\nu g(x)}, \quad \forall \nu \in(0,+\infty), x \in(0,1), \\
\left|J_{\nu}^{\prime}(\nu x)\right| & <\frac{\left(1+x^{2}\right)^{1 / 4} \mathrm{e}^{\nu g(x)}}{x \sqrt{2 \pi \nu}}, \quad \forall \nu \in(0,+\infty), x \in(0,1), \\
J_{\nu}(\nu) & \underset{\nu \rightarrow+\infty}{\sim} \frac{a}{\nu^{1 / 3}},
\end{aligned}
$$

where

$$
g(x):=\ln (x)+\sqrt{1-x^{2}}-\ln \left[1+\sqrt{1-x^{2}}\right] \quad \text { and } \quad a:=\frac{2^{1 / 3}}{3^{2 / 3} \Gamma(2 / 3)}>0 .
$$

Inequalities (4.1) and (4.2) are proved in [43]; inequality (4.3) is in ([1], formula 9.3.31, p. 368). Note that $g$ is negative and increasing on $(0,1)$ and that $g(1)=0$.

Proof of Proposition 4.1. We recall that, in coordinates $\left(r, x^{\prime}\right)$, the Dirichlet-Laplacian writes

$$
\left(-\Delta_{\Omega_{1}}^{D}\right) \varphi=-\frac{\partial^{2} \varphi}{\partial r^{2}}-\frac{d-1}{r} \frac{\partial \varphi}{\partial r}+\frac{1}{r^{2}}\left(-\Delta_{U}^{D}\right) \varphi .
$$

Let $\left(\lambda_{k}^{\prime}\right)_{k \in \mathbb{N}^{*}}$ be the increasing sequence of eigenvalues of $\left(-\Delta_{U}^{D}\right)$ and $\left(X_{k}\right)_{k \in \mathbb{N}^{*}}$ be associated eigenfunctions

$$
\begin{cases}\left(-\Delta_{U}^{D}\right) X_{k}\left(x^{\prime}\right)=\lambda_{k}^{\prime} X_{k}\left(x^{\prime}\right), & x^{\prime} \in U, \\ X_{k}\left(x^{\prime}\right)=0, & x^{\prime} \in \partial U \\ \left\|X_{k}\right\|_{L^{2}(U)}=1 . & \end{cases}
$$

For $k \in \mathbb{N}^{*}$, we define

$$
\nu_{k}:=\sqrt{\lambda_{k}^{\prime}+\left(\frac{d}{2}-1\right)^{2}}
$$


and $j_{k}$ the first positive zero of the Bessel function of first kind $J_{\nu_{k}}$. Note that

$$
\nu_{k}<j_{k}<\nu_{k}+\delta \nu_{k}^{1 / 3}, \quad \forall k \in \mathbb{N}^{*},
$$

for some constant $\delta>0$ (see [1], Formula 9.5.14, p. 371). Let

$$
C_{k}:=\left(\int_{0}^{1}\left|r^{-\frac{d}{2}+1} J_{\nu_{k}}\left(j_{k} r\right)\right|^{2} r^{d-1} \mathrm{~d} r\right)^{1 / 2}, \quad \forall k \in \mathbb{N}^{*} .
$$

Then, for every $k \in \mathbb{N}^{*}$, the function

$$
\widetilde{\varphi}_{k}\left(r x^{\prime}\right):=\frac{1}{C_{k}} r^{-\frac{d}{2}+1} J_{\nu_{k}}\left(j_{k} r\right) X_{k}\left(x^{\prime}\right), \forall r \in(0,1), x^{\prime} \in U,
$$

is a normalized eigenfunction of $\left(-\Delta_{\Omega_{1}}^{D}\right)$ associated to the eigenvalue

$$
\widetilde{\lambda}_{k}:=j_{k}^{2}
$$

Step 1. We prove the existence of $\mathcal{C}_{1}>0$ such that, for $k$ large enough

$$
C_{k} \geqslant \frac{\mathcal{C}_{1}}{\nu_{k}^{3 / 4}}
$$

Let $\epsilon \in(0,5 / 6)$. Performing changes of variables, we get, for $k$ large enough

$$
\begin{aligned}
C_{k} & =\left(\int_{0}^{1}\left|J_{\nu_{k}}\left(j_{k} r\right)\right|^{2} r \mathrm{~d} r\right)^{1 / 2} \\
& =\frac{1}{j_{k}}\left(\int_{0}^{j_{k}}\left|J_{\nu_{k}}(\rho)\right|^{2} \rho \mathrm{d} \rho\right)^{1 / 2} \\
& \geqslant \frac{1}{j_{k}}\left(\int_{0}^{\nu_{k}}\left|J_{\nu_{k}}(\rho)\right|^{2} \rho \mathrm{d} \rho\right)^{1 / 2} \text { by (4.4) } \\
& \geqslant \frac{\nu_{k}}{j_{k}}\left(\int_{0}^{1}\left|J_{\nu_{k}}\left(\nu_{k} r\right)\right|^{2} r \mathrm{~d} r\right)^{1 / 2} \\
& \geqslant C\left(\int_{1-\nu_{k}^{-\frac{5}{6}-\epsilon}}^{1}\left|J_{\nu_{k}}\left(\nu_{k} r\right)\right|^{2} \mathrm{~d} r\right)^{1 / 2} \text { by (4.4). }
\end{aligned}
$$

For $r \in\left(1-\nu^{-\frac{5}{6}-\epsilon}, 1\right)$ and $\nu$ large enough, we have

$$
\begin{aligned}
\left|J_{\nu}(\nu r)\right| & \geqslant\left|J_{\nu}(\nu)\right|-\nu(1-r) \sup \left\{\left|J_{\nu}^{\prime}(\nu \sigma)\right| ; \sigma \in(r, 1)\right\} \\
& \geqslant \frac{a}{2 \nu^{1 / 3}}-\nu^{1-\frac{5}{6}-\epsilon} \frac{C}{\sqrt{\nu}} \quad \text { by }(4.2) \text { and }(4.3) \\
& \geqslant \frac{1}{\nu^{1 / 3}}\left(\frac{a}{2}-\frac{C}{\nu^{\epsilon}}\right) \\
& \geqslant \frac{a}{4 \nu^{1 / 3}} .
\end{aligned}
$$


We deduce from (4.7) and (4.8) that (4.6) holds for some constant $\mathcal{C}_{1}>0$.

Step 2. Conclusion:

Let $\omega_{1}$ be an open subset of $\mathbb{R}^{d}$ such that $\overline{\omega_{1}} \subset \Omega_{1}$. There exists $a \in(0,1)$ such that

$$
\omega_{1} \subset\left\{x=r x^{\prime} ; 0<r<a, x^{\prime} \in U\right\} .
$$

Thus, for every $k \in \mathbb{N}^{*}$,

$$
\begin{aligned}
\int_{\omega_{1}}\left|\widetilde{\varphi}_{k}(x)\right|^{2} \mathrm{~d} x & \leqslant \int_{0}^{a}\left|\frac{1}{C_{k}} r^{-\frac{d}{2}+1} J_{\nu_{k}}\left(j_{k} r\right)\right|^{2} r^{d-1} \mathrm{~d} r \\
& \leqslant \frac{a^{2}}{2 C_{k}^{2}} \sup \left\{J_{\nu_{k}}\left(j_{k} r\right) ; 0<r<a\right\} .
\end{aligned}
$$

Let $b \in(a, 1)$. By (4.4), we have $\frac{j_{k} a}{\nu_{k}}<b<1$ for $k$ large enough. Then, by (4.1) for every $r \in(0, a)$,

$$
0<J_{\nu_{k}}\left(j_{k} r\right)=J_{\nu_{k}}\left(\nu_{k} \frac{j_{k} r}{\nu_{k}}\right) \leqslant \mathrm{e}^{\nu_{k} g\left(\frac{j_{k} r}{\nu_{k}}\right)} .
$$

Explicit computations show that $g^{\prime}(x)>0$, for every $x \in(0,1)$, thus

$$
g\left(\frac{j_{k} r}{\nu_{k}}\right)<g(b)<0, \quad \forall r \in(0, a)
$$

Therefore,

$$
\int_{\omega_{1}}\left|\widetilde{\varphi}_{k}(x)\right|^{2} \mathrm{~d} x \leqslant \frac{a^{2}}{2 C_{k}^{2}} \mathrm{e}^{-|g(b)| \nu_{k}} .
$$

By (4.6), (4.4) and (4.5), we get the conclusion.

Finally, let us quote, without proof, other examples of pairs $\left(\Omega_{1}, \omega_{1}\right)$ satisfying property $\mathcal{P}(s)$ for appropriate values of $s$.

If $\Omega_{1}$ is a filled ellipse and $\omega_{1}$ is an open subset of $\Omega_{1}$ that does not intersect $\partial \Omega_{1}$, then the pair $\left(\Omega_{1}, \omega_{1}\right)$ satisfies property $\mathcal{P}(s)$ for any $s \in(0,1 / 2)$. This can be proved by working in separate variables as in [38] and constructing "whispery galleries" solutions. The same result holds if $\omega_{1}$ intersects $\partial \Omega_{1}$ but does not intersect the small axis of $\Omega_{1}$ (see [38], Thm. 3.1, p. 786). This time this corresponds to "focusing solutions".

All these results can be proved with semi-classical analysis (see, for instance [44] and [23]).

\section{Well POSEDNESS AND Fourier DECOMPOSITION}

In this section $\gamma \in \mathbb{N}^{*}$ and $\beta \in(0,1)$ are fixed. For $f \in C_{c}^{\infty}(\Omega, \mathbb{C})$, we define

$$
|f|_{V}:=\left(\int_{\Omega}\left|\partial_{v} f(x, v)\right|^{2} \mathrm{~d} x \mathrm{~d} v\right)^{1 / 2}
$$

and

$$
V:=\operatorname{Adh}_{|\cdot|_{V}}\left[C_{c}^{\infty}(\Omega, \mathbb{C})\right] .
$$

Observe that $H_{0}^{1}(\Omega) \subset V \subset L^{2}(\Omega)$, thus $V$ is dense in $L^{2}(\Omega)$. We define the operator $A_{\gamma, \beta}$ by

$$
\begin{aligned}
D\left(A_{\gamma, \beta}\right) & :=\left\{f \in V ;-\partial_{v}^{2} f+i v^{\gamma}\left(-\Delta_{x}\right)^{\beta} f \in L^{2}(\Omega)\right\}, \\
A_{\gamma, \beta} f & :=-\partial_{v}^{2} f+i v^{\gamma}\left(-\Delta_{x}\right)^{\beta} f .
\end{aligned}
$$


Then $D\left(A_{\gamma, \beta}\right)$ is dense in $L^{2}(\Omega),\left(A_{\gamma, \beta}, D\left(A_{\gamma, \beta}\right)\right)$ is a closed operator and both $A_{\gamma, \beta}$ and $A_{\gamma, \beta}^{*}$ are dissipative, thus $\left(A_{\gamma, \beta}, D\left(A_{\gamma, \beta}\right)\right)$ generates a strongly continuous semigroup of contractions of $L^{2}(\Omega)$ (see the Lumer-Phillips Theorem [40], Cor. 4.4, Chap. 1, p. 15; or the Hille Yosida's Theorem [12], Thm. VII.4, p. 105).

We consider a solution $g \in C^{0}\left([0, T], L^{2}(\Omega)\right)$ of (1.3). Then, the function $x \mapsto g(t, x, v)$ belongs to $L^{2}\left(\Omega_{1}\right)$ for almost every $(t, v) \in[0,+\infty) \times(-1,1)$, thus, it can be developed on the Hilbert basis $\left(\varphi_{n}\right)_{n \in \mathbb{N}^{*}}($ see $(1.4))$ as follows

$$
g(t, x, v)=\sum_{n \in \mathbb{N}^{*}} g_{n}(t, v) \varphi_{n}(x) \quad \text { where } \quad g_{n}(t, v):=\int_{\Omega_{1}} g(t, x, v) \varphi_{n}(x) \mathrm{d} x, \quad \forall n \in \mathbb{N}^{*} .
$$

In what follows, with a slight abuse of vocabulary, this decomposition is called 'Fourier decomposition' and the functions $g_{n}(t, v)$ are called 'Fourier components'.

Proposition 5.1. For every $n \in \mathbb{N}^{*}, g_{n}$ is the unique solution of

$$
\left\{\begin{array}{lc}
\partial_{t} g_{n}(t, v)+i \lambda_{n}^{\beta} v^{\gamma} g_{n}(t, v)-\partial_{v}^{2} g_{n}(t, v)=0,(t, v) \in(0,+\infty) \times(-1,1), \\
g_{n}(t, \pm 1)=0, & t \in(0,+\infty), \\
g_{n}(0, v)=g_{0, n}(v), & v \in(-1,1),
\end{array}\right.
$$

where $g_{0, n} \in L^{2}(-1,1)$ is given by

$$
g_{0, n}(v):=\int_{\Omega_{1}} g_{0}(x, v) \varphi_{n}(x) \mathrm{d} x, \quad v \in(-1,1) .
$$

This result can be proved by following the same steps as in ([10], Sect. 2.2).

\section{OBservability ON A HORIZONTAL STRIP}

The goal of this section is the proof of the statements 1 of Theorems 1.6 and 1.7. Note that the negative part of the first statement of Theorem 1.7 (i.e. no null controllability, when $\gamma=2$ and $T<T^{*}$ ) can be done exactly as in $[9]$.

\subsection{Global Carleman estimate}

The goal of this subsection is the statement of a global Carleman estimate, proved in ([9], Appendix) and useful for the proof of the statements 1 of Theorems 1.6 and 1.7. For $\lambda \in \mathbb{R}$ and $\gamma \in\{1,2\}$, we introduce the operator

$$
\mathcal{P}_{\lambda, \gamma} g:=\partial_{t} g+i \lambda v^{\gamma} g-\partial_{v}^{2} g .
$$

Proposition 6.1. Let $a, b$ be such that $-1<a<b<1$. There exist a weight function $B \in C^{1}\left([-1,1], \mathbb{R}_{+}^{*}\right)$, positive constants $\mathcal{C}_{1}, \mathcal{C}_{2}$ such that, for every $\lambda \in \mathbb{R}, \gamma \in\{1,2\}, T>0$ and $g \in C^{0}\left([0, T], L^{2}(-1,1)\right) \cap$ $L^{2}\left(0, T ; H_{0}^{1}(-1,1)\right)$ the following inequality holds

$$
\begin{aligned}
\mathcal{C}_{1} \int_{0}^{T} \int_{-1}^{1}\left(\frac{M}{t(T-t)} \mid\right. & \left.\left.\frac{\partial g}{\partial v}(t, v)\right|^{2}+\frac{M^{3}}{(t(T-t))^{3}}|g(t, v)|^{2}\right) \mathrm{e}^{-\frac{M B(v)}{t(T-t)}} \mathrm{d} v \mathrm{~d} t \\
& \leqslant \int_{0}^{T} \int_{-1}^{1}\left|\mathcal{P}_{\lambda, \gamma} g(t, v)\right|^{2} \mathrm{e}^{-\frac{M B(v)}{t(T-t)}} \mathrm{d} v \mathrm{~d} t+\int_{0}^{T} \int_{a}^{b} \frac{M^{3}}{(t(T-t))^{3}}|g(t, v)|^{2} \mathrm{e}^{-\frac{M B(v)}{t(T-t)}} \mathrm{d} v \mathrm{~d} t,
\end{aligned}
$$

where $M:=\mathcal{C}_{2} \max \left\{T+T^{2} ; \sqrt{|\lambda|} T^{2}\right\}$.

In this proposition, the weight $B$ is the usual one for Carleman estimates for $1 \mathrm{D}$ heat equations; since its explicit expression will not be used in this article, we do not specify its properties. Note that we have sharp dependency of $M$ on $\lambda$ and $T$. In particular, if we treat the term $i \lambda v^{\gamma} g$ as a lower-order term, to apply the Carleman estimate for the operator $\left(\partial_{t}-\partial_{v}^{2}\right)$, then, we can obtain a less sharp dependency $M=O\left(\lambda^{2 / 3}\right)$, which is not sufficient in this article. 


\subsection{Dissipation of Fourier components}

The Dirichlet realization of the operator $-\partial_{v}^{2}+i \lambda_{n}^{\beta} v^{\gamma}$ on $(-1,1)$ is not a normal operator. Thus it is not obvious that the exponential decay of the solutions of (5.2) is given by the smallest real part of the eigenvalues of this operator. This question is answered in the following statement.

Proposition 6.2. Let $\gamma \in\{1,2\}$ and

$$
d:=\frac{2 \gamma \beta}{2+\gamma}
$$

There exist $K, \delta>0$ such that, for every $n \in \mathbb{N}^{*}$ and $g_{0, n} \in L^{2}(-1,1)$, the solution of (5.2) satisfies

$$
\left\|g_{n}(t)\right\|_{L^{2}(-1,1)} \leqslant K \mathrm{e}^{-\delta \lambda_{n}^{d} t}\left\|g_{0, n}\right\|_{L^{2}(-1,1)}, \quad \forall t>0 .
$$

Moreover, for every $\epsilon>0$, there exists $n_{*}>0$ such that, for every $n>n_{*},(6.2)$ holds with $K=K_{\epsilon}$ and

$$
\delta=\left\{\begin{array}{l}
\left|\mu_{1}\right| / 2-\varepsilon \text { if } \gamma=1, \\
\sqrt{2} / 2-\varepsilon \text { if } \gamma=2,
\end{array}\right.
$$

where $\mu_{1}$ is the first zero (from the right) of the Airy function.

Finally, the exponent $d$ of $\lambda_{n}$ in (6.2) is optimal, and the critical value of $\delta$ in (6.3) is also optimal.

This result is stronger than ([9], Props. 10 and 17) because in (6.2), we have $L^{2}$-norms on both sides, whereas in [9] there was an $H^{1}$-norm on the right hand side. We study this problem in semi-classical formulation (take $h \leftarrow \lambda_{n}^{-\beta / 2}$ and $\left.t \leftarrow h_{n} t\right)$.

Let $h_{0}>0$. For $h \in\left(0, h_{0}\right)$ and $\psi_{0, h} \in L^{2}(-1,1)$, we consider the equation

$$
\left\{\begin{array}{lc}
h \partial_{t} \psi_{h}(t, v)-h^{2} \partial_{v}^{2} \psi_{h}(t, v)+i v^{\gamma} \psi_{h}(t, v)=0, & (t, v) \in(0,+\infty) \times(-1,1), \\
\psi_{h}(t, \pm 1)=0, & t \in(0,+\infty), \\
\psi_{h}(0, v)=\psi_{0, h}(v), & v \in(-1,1) .
\end{array}\right.
$$

Proposition 6.3. Let $e=2 \gamma /(\gamma+2)$. There exist $K, \delta>0$ such that, for every $h \in\left(0, h_{0}\right)$ and $\psi_{0, h} \in L^{2}(-1,1)$, the unique solution of (6.4) satisfies

$$
\left\|\psi_{h}(t)\right\|_{L^{2}(-1,1)} \leq K \mathrm{e}^{-\delta h^{e-1} t}\left\|\psi_{0, h}\right\|_{L^{2}(-1,1)}, \quad \forall t>0 .
$$

Moreover, for every $\varepsilon>0$, there exists $h^{*} \in\left(0, h_{0}\right)$ such that, for every $h \in\left(0, h^{*}\right)$, (6.5) holds with $K=K_{\varepsilon}$ and (6.3) where $\mu_{1}$ is the first zero (from the right) of the Airy function.

Finally, the exponent $d$ of $h$ in (6.5) is optimal, and the critical value of $\delta$ in (6.3) is also optimal.

Proof of Proposition 6.3.

Let $A_{h}$ be the operator defined by

$$
A_{h}=-h^{2} \frac{\mathrm{d}^{2}}{\mathrm{~d} v^{2}}+i v^{\gamma}, \quad \mathcal{D}\left(A_{h}\right)=H^{2}(-1,1) \cap H_{0}^{1}(-1,1) .
$$

By rescaling $\left(R=R(h)=h^{-e / \gamma}\right.$ and $\left.y=R v\right)$ and using Theorems 3.1 and 3.2, we have

$$
\lim _{h \rightarrow 0} h^{-e} \inf \operatorname{Re} \sigma\left(\mathcal{A}_{h}\right)= \begin{cases}\left|\mu_{1}\right| / 2 & \text { if } \gamma=1, \\ \sqrt{2} / 2 & \text { if } \gamma=2 .\end{cases}
$$


Thus, we can consider

$$
\delta^{*}:=\min _{h \in\left(0, h_{0}\right)} h^{-e} \inf \operatorname{Re} \sigma\left(\mathcal{A}_{h}\right)>0 .
$$

Let $\delta \in\left(0, \delta^{*}\right)$. By Theorems 3.1 and 3.2 , there exists $C_{\delta}$ such that

$$
\sup _{\nu \in \mathbb{R}}\left\|\left(A_{h}-\delta h^{e}-i \nu\right)^{-1}\right\| \leqslant \frac{C_{\delta}}{h^{e}}
$$

Thus,

$$
\sup _{\nu \in \mathbb{R}}\left\|\left(\frac{A_{h}}{h}-\delta h^{e-1}-i \nu\right)^{-1}\right\| \leqslant C_{\delta} h^{1-e} .
$$

Moreover, the operator $h^{-1} A_{h}$ is maximally accretive, thus it generates a semigroup of contractions:

$$
\left\|\psi_{h}(t)\right\|_{L^{2}(-1,1)} \leqslant\left\|\psi_{0, h}\right\|_{L^{2}(-1,1)}, \quad \forall t>0 .
$$

We can apply ([29], Thm. 1.5), with $\omega=-\delta h^{e-1}<0, r(\omega)^{-1} \leq C_{\delta} h^{1-e}, m(t) \equiv 1$ and $a=\tilde{a}=t / 2$. Note that

$$
\|\mathbf{1}\|_{L^{2}\left((0, t / 2) ; \mathrm{e}^{\omega t} \mathrm{~d} t\right)}^{2}=\frac{1-\mathrm{e}^{\omega t / 2}}{-\omega} .
$$

Thus, we obtain

$$
\left\|\psi_{h}(t, \cdot)\right\|_{L^{2}(-1,1)} \leqslant \frac{\delta C_{\delta}}{1-\mathrm{e}^{-\delta h^{e-1} t / 2}} \mathrm{e}^{-\delta h^{e-1} t}\left\|\psi_{0, h}\right\|_{L^{2}(-1,1)}, \quad \forall t>0 .
$$

Let $c_{0}>0$ and $t_{h}=2 c_{0} h^{1-e} / \delta$. Then, by (6.9),

$$
\left\|\psi_{h}(t, \cdot)\right\|_{L^{2}(-1,1)} \leqslant K_{1} \mathrm{e}^{-\delta h^{e-1} t}\left\|\psi_{0, h}\right\|_{L^{2}(-1,1)}, \quad \forall t \geq t_{h}
$$

with

$$
K_{1}=\frac{\delta C_{\delta}}{1-\mathrm{e}^{-c_{0}}}
$$

Moreover, by (6.8),

$$
\left\|\psi_{h}(t)\right\|_{L^{2}(-1,1)} \leqslant K_{2} \mathrm{e}^{-\delta h^{e-1} t}\left\|\psi_{0, h}\right\|_{L^{2}(-1,1)}, \quad \forall t \leq t_{h}
$$

with $K_{2}=\mathrm{e}^{2 c_{0}}$. Thus,

$$
\left\|\psi_{h}(t)\right\|_{L^{2}(-1,1)} \leqslant K \mathrm{e}^{-\delta h^{e-1} t}\left\|\psi_{0, h}\right\|_{L^{2}(-1,1)}, \quad \forall t>0
$$

with $K=\max \left(K_{1}, K_{2}\right)$.

Finally, if $\varepsilon>0$ is fixed, by (6.6) there exists $h^{*} \in\left(0, h_{0}\right)$ such that all the previous estimates hold for $h \in\left(0, h^{*}\right)$ and $\delta$ as in (6.3). Indeed, we have

$$
\delta<\tilde{\delta}^{*}:=\min _{h \in\left(0, h^{*}\right)} h^{-e} \inf \operatorname{Re} \sigma\left(\mathcal{A}_{h}\right) .
$$

To prove the optimality of exponent $(e-1)$ of $h$ in $(6.5)$, we just consider

$$
\psi_{0, h} \in \operatorname{ker}\left(A_{h}-\lambda_{0, h} h^{e}\right) \text {, }
$$

where $\lambda_{0, h}$ satisfies $h^{e} \lambda_{0, h} \in \sigma\left(\mathcal{A}_{h}\right)$ and $h^{e} \operatorname{Re} \lambda_{0, h}=\inf \operatorname{Re} \sigma\left(\mathcal{A}_{h}\right)$. Then, we have

$$
\psi_{h}(t, v)=\mathrm{e}^{-\lambda_{0, h} h^{e-1} t} \psi_{0, h}(v) .
$$

Thus, by (6.6), for every $t>0$ and $\varepsilon>0$, there exists $h^{*}>0$ such that, for every $h \in\left(0, h^{*}\right)$,

$$
\begin{aligned}
\left\|\psi_{h}(t, \cdot)\right\|_{L^{2}(-1,1)} & =\mathrm{e}^{-\lambda_{0, h} h^{e-1} t}\left\|g_{0, n}\right\|_{L^{2}(-1,1)} \\
& \geqslant \mathrm{e}^{-(\nu+\varepsilon) h^{e-1} t}\left\|\psi_{0, h}\right\|_{L^{2}(-1,1)},
\end{aligned}
$$

with $\nu=\left|\mu_{1}\right| / 2$ if $\gamma=1$ and $\nu=\sqrt{2} / 2$ if $\gamma=2$. 


\subsection{Proof of the positive statements of Theorems 1.6 and 1.7}

The positive statements in Theorems 1.6 and 1.7 are consequences of the following proposition and of the Bessel-Parseval equality.

Proposition 6.4. Let $\beta \in(0,1)$ and $0<a<b<1$.

- If $\gamma=1$, then, for every $T>0$, there exists $C>0$ such that for every $n \in \mathbb{N}^{*}$ and $g_{0, n} \in L^{2}(-1,1)$, the solution of (5.2) satisfies

$$
\int_{-1}^{1}\left|g_{n}(T, v)\right|^{2} \mathrm{~d} v \leqslant C \int_{0}^{T} \int_{a}^{b}\left|g_{n}(t, v)\right|^{2} \mathrm{~d} v \mathrm{~d} t .
$$

- If $\gamma=2$, then, there exists $T_{1}>0$ such that, for every $T>T_{1}$, there exists $C>0$ such that for every $n \in \mathbb{N}^{*}$ and $g_{0, n} \in L^{2}(-1,1)$, the solution of $(5.2)$ satisfies $(6.11)$.

Proof of Proposition 6.4.

We deduce from Proposition 6.1 that

$$
\mathcal{C}_{3} \lambda_{n}^{3 \beta / 2} \mathrm{e}^{-c^{*} \lambda_{n}^{\beta / 2}} \int_{T / 3}^{2 T / 3} \int_{-1}^{1}\left|g_{n}(t, v)\right|^{2} \mathrm{~d} v \mathrm{~d} t \leqslant \mathcal{C}_{4} \int_{0}^{T} \int_{a}^{b}\left|g_{n}(t, v)\right|^{2} \mathrm{~d} v \mathrm{~d} t
$$

for $n$ large enough, where $\mathcal{C}_{3}:=\mathcal{C}_{2} \max \left\{4 \mathcal{C}_{1} ;\left(4 \mathcal{C}_{1}\right)^{3}\right\}, c^{*}:=\frac{9}{2} \mathcal{C}_{2} \max \{\beta(v) ; v \in[-1,1]\}, \mathcal{C}_{4}:=\max \left\{x^{3} \mathrm{e}^{-\beta_{*} x} ; x \geqslant\right.$ $0\}$ and $\beta_{*}:=\min \{\beta(v) ; v \in(a, b)\}$.

Moreover, thanks to Proposition 6.2, we have

$$
\begin{aligned}
\int_{-1}^{1}\left|g_{n}(T, v)\right|^{2} \mathrm{~d} v & \leqslant \frac{3 K^{2}}{T} \mathrm{e}^{-2 \delta \lambda_{n}^{d} T / 3} \int_{T / 3}^{2 T / 3} \int_{-1}^{1}\left|g_{n}(t, v)\right|^{2} \mathrm{~d} v \mathrm{~d} t \\
& \leqslant \frac{\mathcal{C}_{5}}{\lambda_{n}^{3 \beta / 2}} \mathrm{e}^{c^{*} \lambda_{n}^{\beta / 2}-2 \delta \lambda_{n}^{d} T / 3} \int_{0}^{T} \int_{a}^{b}\left|g_{n}(t, v)\right|^{2} \mathrm{~d} v \mathrm{~d} t
\end{aligned}
$$

where $\mathcal{C}_{5}:=K^{2} \mathcal{C}_{4} / \mathcal{C}_{3}$

Case 1: $\gamma=1$. Then $d=\frac{2 \beta}{3}>\frac{\beta}{2}$, thus the observability constant above converges to zero as $n \rightarrow+\infty$. This proves the existence of a uniform observability constant for high frequencies: there exists $\mathcal{C}_{H}>0$ and $n_{0} \in \mathbb{N}^{*}$ such that

$$
\int_{-1}^{1}\left|g_{n}(T, v)\right|^{2} \mathrm{~d} v \leqslant \mathcal{C}_{H} \int_{0}^{T} \int_{a}^{b}\left|g_{n}(t, v)\right|^{2} \mathrm{~d} v \mathrm{~d} t, \quad \forall g_{n}^{0} \in L^{2}(-1,1), n>n_{0} .
$$

Moreover, for every $n \in\left\{1, \ldots, n_{0}\right\}$, there exists a constant $C_{n}>0$ such that

$$
\int_{-1}^{1}\left|g_{n}(T, v)\right|^{2} \mathrm{~d} v \leqslant C_{n} \int_{0}^{T} \int_{a}^{b}\left|g_{n}(t, v)\right|^{2} \mathrm{~d} v \mathrm{~d} t, \quad \forall g_{n}^{0} \in L^{2}(-1,1)
$$

(usual observability inequality for 1D heat equations). Thus, the uniform observability constant $C:=\max \left\{\mathcal{C}_{H}, C_{n} ; 1 \leqslant n \leqslant n_{0}\right\}$ gives the conclusion.

Case 2: $\gamma=2$. Then $d=\frac{\beta}{2}$, thus, when $T>T_{1}:=\frac{3 c_{*}}{2 \delta}$, the observability constant in (6.13) converges to zero as $n \rightarrow+\infty$ and the proof can be ended as in the previous case. 


\section{REFERENCES}

[1] M. Abramowitz and I.A. Stegun, Handbook of mathematical functions with formulas graphs and mathematical tables. Edited by Milton. New York, Dover (1972).

[2] F. Alabau-Boussouira, P. Cannarsa, and G. Fragnelli, Carleman estimates for degenerate parabolic operators with applications to null controllability. J. Evol. Equ. 6 (2006) 161-204.

[3] S. Alinhac and C. Zuily, Uniqueness and nonuniqueness of the Cauchy problem for hyperbolic operators with double characteristics. Commun. Partial Differ. Equ. 6 (1981) 799-828.

[4] Y. Almog, The stability of the normal state of superconductors in the presence of electric currents. Siam J. Math. Anal. 40 (2008) 824-850.

[5] Y. Almog and B. Helffer, Global stability of the normal state of superconductors in the presence of a strong electric current. Commun. Math. Phys. 330 (2014) 1021-1094.

[6] Y. Almog, B. Helffer and X. Pan, Superconductivity near the normal state in a half-plane under the action of a perpendicular electric current and an induced magnetic field II: The large conductivity limit. SIAM J. Math. Anal. 44 (2012) 3671-3733.

[7] Y. Almog, B. Helffer and X. Pan, Superconductivity near the normal state in a half-plane under the action of a perpendicular electric current and an induced magnetic field. Trans. Amer. Math. Soc. 365 (2013) 1183-1217.

[8] Y. Almog, B. Helffer and X.-B. Pan, Superconductivity near the normal state under the action of electric currents and induced magnetic fields in $\mathbb{R}^{2}$. Commun. Math. Phys. 300 (2010) 147-184.

[9] K. Beauchard. Null controllability of Kolmogorov-type equations. Math. Control Signals Syst. 26 (2014) $145-176$.

[10] K. Beauchard, P. Cannarsa and R. Guglielmi. Some controllability results for the 2D Grushin equations. J. Eur. Math. Soc. 16 (2014) 67-101.

[11] J.-M. Bony, Principe du maximum, inégalité de Harnack et unicité du problème de Cauchy pour les opérateurs elliptiques dégénérés. Ann. Inst. Fourier 19 (1969) 277-304.

[12] H. Brézis, Analyse Fonctionnelle, Théorie et Applications. Masson, Paris (1983).

[13] J.-M. Buchot and J.-P. Raymond, Feedback stabilization of a boundary layer equation, part2: Nonhomogeneous state equations and numerical simulations. Appl. Math. Res. Express 2009 (2010) 877-122.

[14] J.-M. Buchot and J.-P. Raymond, Feedback stabilization of a boundary layer equation, part 1. ESAIM:COCV 17 (2011) $506-551$.

[15] P. Cannarsa and L. de Teresa, Controllability of 1-D coupled degenerate parabolic equations. Electron. J. Differ. Equ. 73 (2009) 21.

[16] P. Cannarsa, G. Fragnelli and D. Rocchetti, Null controllability of degenerate parabolic operators with drift. Netw. Heterog. Media 2 (2007) 695-715.

[17] P. Cannarsa, G. Fragnelli and D. Rocchetti, Controllability results for a class of one-dimensional degenerate parabolic problems in nondivergence form. J. Evol. Equ. 8 (2008) 583-616.

[18] P. Cannarsa, P. Martinez and J. Vancostenoble, Persistent regional null controllability for a class of degenerate parabolic equations. Commun. Pure Appl. Anal. 3 (2004) 607-635.

[19] P. Cannarsa, P. Martinez and J. Vancostenoble, Null controllability of degenerate heat equations. Adv. Differ. Equ. 10 (2005) $153-190$.

[20] P. Cannarsa, P. Martinez and J. Vancostenoble, Carleman estimates for a class of degenerate parabolic operators. SIAM J. Control Optim. 47 (2008) 1-19.

[21] P. Cannarsa, P. Martinez and J. Vancostenoble, Carleman estimates and null controllability for boundary-degenerate parabolic operators. C. R. Math. Acad. Sci. Paris 347 (2009) 147-152.

[22] E.B. Davies, Wild spectral behaviour of anharmonic oscillators. Bull. London Math. Soc. 32 (2000) 432-438.

[23] S. Didelot, Etude d'une perturbation singulière elliptique dégénérée. Thèse de doctorat, Reims (1999).

[24] H.O. Fattorini and D. Russel, Exact controllability theorems for linear parabolic equations in one space dimension. Arch. Ration. Mech. Anal. 43 (1971) 272-292.

[25] C. Flores and L. de Teresa, Carleman estimates for degenerate parabolic equations with first order terms and applications. $C$. R. Math. Acad. Sci. Paris 348 (2010) 391-396.

[26] A.V. Fursikov and O.Y. Imanuvilov, Controllability of evolution equations. Vol. 34 of Lect. Notes Series. Seoul National University Research Institute of Mathematics Global Analysis Research Center, Seoul (1996).

[27] B. Helffer, Spectral Theory and its Applications. Cambridge University Press (2013).

[28] B. Helffer and D. Robert, Propriétés asymptotiques du spectre d'opérateurs pseudo-différentiels sur $\mathbb{R}^{n}$. Commun. Partial Differ. Eq. 7 (1982) 795-882.

[29] B. Helffer and J. Sjöstrand, From resolvent bounds to semigroup bounds, Appendix of a course by Sjöstrand. Proc. of the Evian Conference (2009). Preprint arXiv: 1001.4171

[30] R. Henry, On the semi-classical analysis of Schrödinger operators with purely imaginary electric potentials in a bounded domain. Preprint arXiv: 1405.6183

[31] O.Y. Imanuvilov, Boundary controllability of parabolic equations. Uspekhi. Mat. Nauk 48 (1993) 211-212.

[32] O.Y. Imanuvilov, Controllability of parabolic equations. Mat. Sb. 186 (1995) 109-132.

[33] T. Kato, Perturbation Theory for Linear operators. Springer-Verlag, Berlin New-York (1966).

[34] G. Lebeau and L. Robbiano, Contrôle exact de l'équation de la chaleur. Commun. Partial Differ. Eq. 20 (1995) 335-356. 
[35] G. Lebeau and J. Le Rousseau, On Carleman estimates for elliptic and parabolic operators. Applications to unique continuation and control of parabolic equations. ESAIM:COCV 18 (2012) 712-747.

[36] P. Martinez and J. Vancostenoble, Carleman estimates for one-dimensional degenerate heat equations. J. Evol. Equ. 6 (2006) $325-362$.

[37] P. Martinez, J. Vancostonoble and J.-P. Raymond, Regional null controllability of a linearized Crocco type equation. SIAM J. Control Optim. 42 (2003) 709-728.

[38] B.-T. Nguyen and D.S. Grebekov, Localization of laplacian eigenfunctions in circular and elliptical domains. SIAM J. Appl. Math. 73 780-803.

[39] O.A. Oleinik and V.N. Samokhin, Mathematical Models in Boundary Layer Theory. In vol. 15 of Appl. Math. Math. Comput. Chapman Hall CRC, Boca Raton, London, New York (1999).

[40] A. Pazy, Semigroups of linear operators and applications to partial differential equations. Appl. Math. Sci. Springer Verlag, New-York (1983).

[41] K. Pravda-Starov, A complete study of the pseudo-spectrum for the rotated harmonic oscillator. J. London Math. Soc. 73 (2006) 745-761.

[42] Y. Sibuya, Global theory of a second order linear ordinary differential equation with a polynomial coefficient. Amsterdam, North-Holland (1975).

[43] K.M. Siegel, An inequality involving Bessel functions of argument nearly equal to their orders. Proc. Amer. Math. Soc. 4 (1953) 858-859.

[44] J. Toth and S. Zelditch, Counting nodal lines wich touch the boundary of an analytic domain. J. Differ. Geometry 81 (2009) 649-686. 